\title{
MARTINGALES AND ONE-DIMENSIONAL DIFFUSION
}

BY

J. L. DOOB

1. Introduction. Let $B$ be a Banach space, and let $\left\{U_{t}, t \geqq 0\right\}$ be a family of bounded linear operators taking $B$ into itself. We shall call the family a semigroup if $U_{0}$ is the identity, and if

$$
U_{8+t}=U_{s} U_{t}
$$

The infinitesimal operator of a semigroup is defined by

$$
A f=\underset{h \rightarrow 0}{\operatorname{strong} \lim } \frac{U_{h} f-f}{h}
$$

whenever this limit exists.

A semigroup will be said to be of type $\Gamma$ if the following conditions are satisfied. The space $B$ is the space of continuous functions $f$ on a Hausdorff space satisfying

$$
\|f\|=\sup _{\xi} \frac{|f(\xi)|}{\phi(\xi)}<\infty,
$$

where $\phi$ is continuous, and is bounded from below by a positive number. The operators of the semigroup take non-negative functions into non-negative functions, the constant functions are invariant, and, for every $\delta>0$,

$$
\sup _{\delta<t<1 / \delta}\left\|U_{t}\right\|<\infty .
$$

In our applications we shall never make any assumptions on the continuity of the semigroup considered as a function of the parameter.

The following rather trivial lemma will be useful. Its proof will be omitted.

Lemma 1.1. Let $\left\{U_{t}, 0 \leqq t<\infty\right\}$ be a semigroup of type $\Gamma$. Then if strong $\lim _{n \rightarrow \infty} f_{n}=f \geqq 0$, it follows that $\lim \inf _{n \rightarrow \infty} U_{t_{n}} f_{n} \geqq 0$ whenever $\left\{t_{n}\right\}$ is a bounded sequence of numbers with a positive lower bound.

In our probability discussion, we shall always consider random variables defined on a space $\Omega$ of points $\omega$. The value of an $\Omega$ function $x$ at $\omega$ will be denoted by $x(\omega)$. The general notation of [2] will be used.

Let $\{x(t), t \in T\}$ be a Markov process, with state space the closed set $X$ in $n$-dimensional space, parameter interval $T$, and some specified transition probability function. That is, there is a function $p$, with the arguments $s \in T$,

Received by the editors September 10, 1953. 
$t \in T(s \leqq t), \xi \in X$, and $A$, where $A$ is a Borel subset of $X$, such that, using the notation of [2].

$$
P\{x(t, \omega) \in A \| x(s, \omega)=\xi\}=p(s, t, \xi, A)
$$

with probability 1 . The following hypotheses are made.

M1 For each $s, t, \xi$, the function $p(s, t, \xi, \cdot)$ is a probability measure.

M2 For each $s, t, A$, the function $p(s, t, \cdot, A)$ is a Baire function.

M3

$$
p(t, t, \xi, A)=\left\{\begin{array}{lll}
1 & \text { if } & \xi \in A, \\
0 & \text { if } & \xi \notin A .
\end{array}\right.
$$

M4 The Chapman-Kolmogorov equation is satisfied:

$$
p(s, u, \xi, A)=\int_{X} p(t, u, \eta, A) p(s, t, \xi, d \eta), \quad s \leqq t \leqq u .
$$

In the following, conditional probabilities like that on the left side of (1.1), and corresponding conditional expectations, will be taken as the unique evaluations obtained by use of the function $p$. We shall usually deal with the case of stationary absolute probabilities, in which $p$ can be expressed in terms of a function of one less argument in the form

$$
p(s, t, \xi, A)=p(t-s, \xi, A),
$$

where the quantity on the right defines a function having the properties implied by M1-M4. In this case, if $T=[0, \infty)$, we define an operator $U_{t}$ by

$$
\left(U_{t} f\right)(\xi)=\int_{X} f(\eta) p(t, \xi, d \eta)=E\{f[x(t)] \| x(0, \omega)=\xi\}, \quad t \geqq 0 .
$$

The Banach space domain of the family of operators defined in this way will be specified in $\$ 6$. Obviously $U_{0}$ is the identity, and the fact that $U_{s+t}=U_{s} U_{t}$ is, at least formally, an immediate consequence of the Chapman-Kolmogorov equation. The formal verification becomes rigorous when the domain of $U_{t}$ is specified, and it will then also be clear that the family of operators is of type $\Gamma$. If $g$ is in the domain of the semigroup, and if $a>0$, the stochastic process

$$
\left\{\left(U_{a-t} g\right)[x(t)], 0 \leqq t \leqq a\right\}
$$

is a martingale, if the random variables of this process have finite expectations. In fact, using the Markov property of the $x(t)$ process, we can write

$$
\begin{aligned}
\left(U_{a-t} g\right)[x(t)] & =E\{g[x(a)] \| x(t)\} \\
& =E\{g[x(a)] \| x(s), s \leqq t\},
\end{aligned}
$$

so that the quantity on the left is the conditional expectation of $g[x(a)]$ relative to a family of random variables which increases with $t$. (For definitions 
and a discussion of martingale concepts see [2].) More generally, if $\left\{g_{t}, 0 \leqq t \leqq a\right\}$ is a family of elements in the domain of the semigroup, with

$$
g_{s+t} \leqq U_{t} g_{\text {s }}
$$

the stochastic process

$$
\left\{g_{a-t}[x(t)], 0 \leqq t \leqq a\right\}
$$

is a semimartingale if the random variables have finite expectations. In fact, if $s<t$,

$$
\begin{aligned}
E\left\{g_{a-t}[x(t)] \| x\left(s^{\prime}\right), s^{\prime} \leqq s\right\} & =E\left\{g_{a-t}[x(t)] \| x(s)\right\} \\
& =\left(U_{t-s} g_{a-t}\right)[x(s)] \\
& \geqq g_{a-s}[x(s)] .
\end{aligned}
$$

Leaving the semimartingale case for the moment, this discussion suggests that if $g$ is chosen in such a way that $U_{t} g$ varies in a simple way with $t$, so that (1.3) defines a simple martingale, then the known properties of martingale sample functions will lead to new facts about Markov process sample functions. It is the purpose of this paper to exploit this idea when the $x(t)$ process is a one-dimensional diffusion process. The probability relations of such a process in an open interval are determined by two functions, called the diffusion coefficients, and denoted in this paper by $\sigma^{2}, m$. It is a classical fact that, at least formally, the infinitesimal operator of the $U_{t}$ semigroup becomes a differential operator $D$ given by

$$
D=\frac{\sigma^{2}}{2} \frac{d^{2}}{d \xi^{2}}+m \frac{d}{d \xi}
$$

in the diffusion case.

$\checkmark \mathrm{V}$ e shall choose $g$ in (1.3) in two ways. The first choice will make

$$
U_{t} g=e^{\lambda t} g
$$

the second choice will make

$$
U_{t} g=g+\lambda t,
$$

where $\lambda$ is a constant in each case. The first problem is to identify the functions $g$ with these properties. Obviously $A g=\lambda g$ in the first case, and $A g=\lambda$ in the second. Conversely, if the semigroup is strongly continuous, Hille has shown that $A$ is defined on a dense set in the domain space, and it follows trivially from his work that the equations $A g=\lambda g, A g=\lambda$ imply (1.6) and (1.7) respectively. Thus it would appear that we need only find the characteristic functions of the operator $A$, and the functions taken by $A$ into the constant functions. This is gratifying, in view of the identification of $A$ with the differential operator $D$, but unfortunately we shall not be able to use 
Hille's work, since it seems to be difficult to choose the Banach space of continuous functions as domain space for the semigroup in such a way that the semigroup will be strongly continuous and that simultaneously $A$ can be identified with $D$. We shall, therefore, justify the above reasoning on the application of $A$, by the following rather superficial result, instead of Hille's deep theorems. The advantage of the following theorem is that it involves no hypothesis on the continuity of the semigroup.

THEOREM 1.1. Let $\left\{U_{t}, 0 \leqq t<\infty\right\}$ be a semigroup of type $\Gamma$, with infinitesimal operator $A$. Then

(i) $A g=\lambda g$ implies that $U_{t} g=e^{\lambda t} g, t \geqq 0$, and

(i') $A g=\lambda$ implies that $U_{t} g=g+\lambda t, t \geqq 0$.

(ii) $A g \geqq \lambda g$ implies that $U_{t} g \geqq e^{\lambda t} g, t \geqq 0$, and

(ii') $A g \geqq \lambda$ implies that $U_{t} g \geqq g+\lambda t, t \geqq 0$.

Proof of (i) and (ii). We can suppose that $\lambda=0$ throughout the discussion, replacing $U_{t}$ by $e^{-\lambda t} U_{t}$ otherwise. Under the hypotheses of (i), if $A g=0$ we find that

$$
\underset{h \downarrow 0}{\text { strong } \lim } \frac{U_{t+h} g-U_{t} g}{h}=U_{t} A g=0, \quad t \geqq 0,
$$

since $U_{t}$ is a bounded operator. Moreover

$$
\text { strong } \lim \frac{U_{t-h} g-U_{t} g}{-h}=\underset{h \downarrow 0}{\text { strong }} \lim U_{t-h} \frac{U_{h} g-g}{h}=0, \quad t>0,
$$

since the norm of $U_{t-h}$ has a bound independent of $h$, for small $h$, by hypothesis. Thus $U_{t} g$ defines a function of $t$, with values in the Banach domain space, whose derivative vanishes identically. It follows that $U_{t} g=U_{0} g=g$, as was to be proved. If $A g \geqq 0$, the two preceding limit relations yield, in view of Lemma 1.1, the conclusion that $\left(U_{t} g\right)(\xi)$ defines, for each value of $\xi$, a numerically valued function of $t$ whose derivative on the right exists and is $\geqq 0$, and whose lower derivative on the left is non-negative. It follows that $U_{t} g \geqq U_{0} g=g$, as was to be proved.

The proofs of $\left(\mathrm{i}^{\prime}\right)$ and $\left(\mathrm{ii}^{\prime}\right)$ are similar to those of (i) and (ii), and will be omitted.

It now follows from our discussion that, if $\left\{U_{t}, 0 \leqq t<\infty\right\}$ is a semigroup of type $\Gamma$, defined by (1.2), and if $A g=\lambda g$, then the stochastic process

$$
\left\{e^{-\lambda t} g[x(t)], 0 \leqq t<\infty\right\},
$$

to which (1.3) reduces after a cancellation of a constant factor, and deletion of the now irrelevant $a$, is a martingale. On the other hand, if $A g \geqq \lambda g$, and if we set $g_{t}=e^{\lambda t} g$, we find that (1.4) is satisfied, so that the process (1.8), to which $\left(1.3^{\prime}\right)$ reduces after deletion of irrelevancies, is a semimartingale. 
Finally, if $A g=\lambda$ we find that the stochastic process

$$
\{g[x(t)]-\lambda t, 0 \leqq t<\infty\}
$$

is a martingale, and that if $A g \geqq \lambda$ this process is a semimartingale. All these statements have the common hypothesis that the random variables of the processes have finite expectations.

If the $x(t)$ process is Brownian motion in $n$ dimensions, and if $U_{t}$ is defined by (1.2), the operator $A$ becomes, formally, the Laplacian operator in $n$ dimensions, and our discussion suggests relations between subharmonic and harmonic functions on the one hand, and Brownian motion on the other, to be investigated using martingale theory. In a recent paper [3] the author carried out such an investigation in part, without detailing the above motivation, and in fact without using Banach space notions.

In the present paper the $x(t)$ process will be a one-dimensional diffusion process, and $A$ will be identified with the differential operator $D$ defined above. The critical functions $g$ of (1.8) and (1.9) will thus be readily identifiable. The basic theorems 8.1 and 9.1 are proved in accordance with these ideas, and are used to derive various asymptotic properties of diffusion process sample functions. It has long been obvious to probabilists that these theorems were valid under sufficiently strong hypotheses on the diffusion coefficients. (An exception is the possibility in Theorem 9.6 that condition $A\left(r_{2}\right)$ but not $B\left(r_{2}\right)$ is satisfied, in which case trajectories may approach a barrier asymptotically without reaching it. This is a somewhat unexpected possibility.) However, it has been difficult to treat this problem, because diffusion processes have been defined in terms of the diffusion coefficients in such an indirect fashion that the connection was difficult to follow in a precise way. In this paper we shall use the direct construction of diffusion processes due to Ito ([9], see also [2]) and thus avoid the use of existence theorems of parabolic differential equations. Because of the importance of the Ito processes, we shall prove the required numerical approximations with more generality than is required for this paper.

Doeblin's proofs of his announced [1] partial results in the direction of some of those in this paper were never published. Fortet's treatment of this general subject [7] was devoted to somewhat different problems, and there is essentially no overlap. The most recent, and most detailed, results in this direction were obtained by Feller [6]. Feller was interested in certain semigroup problems, and, as a result, his hypotheses involve restrictions on the diffusion coefficients which are not easy to translate into regularity conditions. His results are less complete in the area of overlap because of the difference in emphasis. The emphasis in the present paper is on a detailed analysis of diffusion process sample functions and absorbing barrier problems. The martingale methods are as adapted to the classical discrete parameter ruin problems as to continuous parameter problems in any number of dimen- 
sions. In the latter application, as soon as a theory of the relevant differential equations is available the corresponding probability theory can be developed along the lines of this paper.

In discussions of this sort, one frequently cannot treat a stochastic process directly, but must deal first with processes derived from it by optional stopping, or, on the other hand, one treats stochastic processes whose state spaces are not the whole space by extending them in such a way that they become processes derived by optional stopping from processes whose state space is the whole space. The concept of optional stopping is treated at length in [2]. The following theorems are useful in such discussions. They were used, in a slightly different form, in [3].

THEOREM 1.2. For each $n \geqq 1$, let $\left\{x_{n}(t), \mathcal{F}(t), t \in T\right\}$ be a semimartingale almost all of whose sample functions are right continuous, and suppose that the $x_{n}(t)$ process is derived from the $x_{n+1}(t)$ process by optional stopping at time $\tau_{n} \leqq b$, with $\tau_{1} \leqq \tau_{2} \leqq \cdots$. Here $T$ is an interval with right-hand end point $b \notin T$. Suppose that

$$
\sup _{n, i} E\left\{\left|z_{n}(t)\right|\right\}<\infty,
$$

and define $z(t)$ by

$$
\lim _{n \rightarrow \infty} z_{n}(t, \omega)= \begin{cases}z(t, \omega) & \text { if } t<\tau(\omega)=\lim _{n \rightarrow \infty} \tau_{n}(\omega), \\ 0 & \text { if } t \geqq \tau(\omega) .\end{cases}
$$

Then $\sup _{t} E\{|z(t)|\}<\infty$, and $\lim _{t \uparrow \tau} z(t)=z$ exists and is finite with probability 1.

The boundedness of the function defined by $E\{|z(t)|\}$ is a consequence of Fatou's lemma. If $\tau_{n}=b$ with probability 1 for each $n$, the $z_{n}(t)$ and $z(t)$ processes are the same, and the existence of the limit $z$ is a standard martingale result. (See Chapter VII, Theorem 4.1.) This martingale result is obtained by showing that the number of oscillations of the $x(t)$ process sample functions, between any specified limits, is almost always bounded. The same proof can be applied here to show that the number of such oscillations of $z_{n}(t)$ process sample functions is almost always bounded, uniformly in the variable $n$. We omit details since the proofs are so similar.

In the following theorem, we need the concept of a semimartingale or martingale relative to a specified family of Borel fields. See [2] for a discussion of this concept.

THEOREM 1.3. For each $n \geqq 1$, let $\left\{z_{n}(t) G_{n}(t), t \in T\right\}$ be a semimartingale [martingale] and suppose that, for each $t, G_{n}(t) \subset G_{n+1}(t), \lim _{n \rightarrow \infty} z_{n}(t)=z(t)$ exists, with probability 1 , and the sequence $\left\{z_{n}(t), n \geqq 1\right\}$ is uniformly integrable. Then the process $\{z(t), t \in T\}$ is a semimartingale [martingale]. 
Let $G_{\infty}(t)$ be the smallest Borel field of $\omega$ sets including all sets of measure 0 and the sets of the field $\bigcup_{1}^{\infty} G_{m}(t)$. Then $z(t)$ is measurable relative to $G_{\infty}(t)$. If $\Lambda \in G_{m}(t)$, and if $s<t$,

$$
\int_{\Lambda} z_{n}(t) d P \geqq \int_{\Lambda} z_{n}(s) d P
$$

by definition of semimartingales (to which we restrict our attention since the proof needs no change in the martingale case), if $n$ is sufficiently large. When $n \rightarrow \infty$, this inequality becomes, in view of the uniform integrability hypothesis,

$$
\int_{\Lambda} z(t) d P \geqq \int_{\Lambda} z(s) d P .
$$

Since this is true for every $\Lambda \in \cup_{1}^{\infty} G_{m}$, it is true for $\Lambda \in G_{\infty}(t)$, and it follows that the $z(t)$ process is a semimartingale (relative to the $G_{\infty}(t)$ family of Borel fields).

Theorem 1.3 will be applied as follows. A $z(t)$ process will be given, whose random variables will be uniformly bounded. The $z_{n}(t)$ process will be derived from it by optional stopping at time $\tau_{n}$, where $\tau_{n} \leqq \tau_{n+1}$. The hypotheses of the theorem will then be trivially satisfied.

2. Standard Ito processes. The diffusion processes to be treated in this paper are based on Markov processes constructed in a way devised by Ito [9]. We shall include the nonstationary case because some of the preliminary results are of independent interest in this case, although the main emphasis will be on the stationary case. The parameter interval $T$ will be assumed, for notational simplicity, to have, and include, the left-hand end point 0 . The interval $T$ may be infinite, and, if finite, may or may not include its righthand end point. Let $m, \sigma$ be Baire functions of the pair $(t, \xi)$ for $0 \leqq t<\infty$, $-\infty<\xi<\infty$. If $T$ is compact, it is supposed that there is a constant $K$ such that

$$
\text { SIP } \begin{array}{cl}
|m(t, \xi)| \leqq K\left(1+\xi^{2}\right)^{1 / 2}, & 0 \leqq \sigma(t, \xi) \leqq K\left(1+\xi^{2}\right)^{1 / 2}, \\
\left|m\left(t, \xi_{2}\right)-m\left(t, \xi_{1}\right)\right| \leqq K\left|\xi_{2}-\xi_{1}\right|, & \left|\sigma\left(t, \xi_{2}\right)-\sigma\left(t, \xi_{1}\right)\right| \leqq K\left|\xi_{2}-\xi_{1}\right| .
\end{array}
$$

If $T$ is not compact, this hypothesis is made on every compact subset of $T$. The constant $K$ may then depend on the compact subset in question.

Let $\{y(t), 0 \leqq t<\infty\}$ be a one-dimensional Brownian motion: $y(0)=0$; the $y(t)$ process is real and Gaussian, with independent increments; it satisfies the equations

$$
E\{y(t)\}=0, \quad E\left\{|y(t)-y(s)|^{2}\right\}=|t-s| ;
$$

almost all its sample functions are continuous. Let $x(0)$ be any random variable defined on the same measure space as the $y(t)$ 's and independent of the 
$y(t)$ process. Ito proved that there is then a Markov process $\{x(t), t \in T\}$, which we shall call a standard Ito process below, which has a transition probability function in the sense of $\$ 1$, whose sample functions are almost all continuous, and which satisfies the symbolic equation

$$
d x(t)=m[t, x(t)] d t+\sigma[t, x(t)] d y(t),
$$

$t \in T$

or, more precisely,

$$
x(t)=x(0)+\int_{0}^{t} m[s, x(s)] d s+\int_{0}^{t} \sigma[s, x(s)] d y(s), \quad t \in T .
$$

In the following, we shall call two stochastic processes $\left\{x_{i}(t), t \in T_{i}\right\}, i=1,2$, essentially the same if they are defined on the same measure space, if $T_{1}=T_{2}$, and if

$$
P\left\{x_{1}(t, \omega)=x_{2}(t, \omega), t \in T_{1}\right\}=1 .
$$

Then the solution of (2.1) is unique in the sense that, if the diffusion coefficients $\sigma^{2}, m$, the $y(t)$ process, and $x(0)$, are specified, two standard Ito processes are essentially the same. If $x(0)$ is specified as the constant initial value $\xi$, we write $x_{\xi}(t)$ for $x(t)$. The transition probability function is uniquely determined by the diffusion coefficients, and in fact we can take

$$
p(0, \xi, t, A)=P\left\{x_{\xi}(t, \omega) \in A\right\},
$$

with a corresponding evaluation of $p(s, \xi, t, A)$ by means of a solution of (2.1) in the interval $[s, \infty) T$.

In particular, if the diffusion coefficients do not depend on the parameter, the transition probability function is stationary, and it is no further restriction on the diffusion coefficients to assume that $T=[0, \infty)$.

Let $\mathcal{F}_{t}$ be the smallest Borel field of $\omega$ sets containing all $\omega$ sets of measure 0 and which has the property that $y(s)$ is measurable relative to the field when $s \leqq t$. Let $\mathcal{F}_{i}^{\prime}$ be the smallest Borel field of $\omega$ sets such that $x(0)$ is measurable with respect to $\mathcal{F}_{\boldsymbol{t}}^{\prime}$ and that $\mathcal{F}_{\boldsymbol{t}}^{\prime} \supset \mathcal{F}_{\boldsymbol{t}}$. Then if $A$ is a linear Borel set, and if $s<t$,

$$
P\left\{x(t, \omega) \in A \| \mathcal{F}_{s}^{\prime}\right\}=P\{x(t, \omega) \in A \| x(s)\}
$$

with probability 1 . This is a slightly stronger condition than the Markov property, and is commonly useful.

We shall need the following evaluations (see [2, p. 285]; the author takes this opportunity to correct (3.18) on that page, which should read as written here)

$$
\begin{aligned}
P\left\{\operatorname{Max}_{t \leqq u \leqq t+h}|x(u, \omega)-\xi|>\epsilon \| x(t, \omega)\right. & =\xi\} \\
& \leqq\left(1+\xi^{2}\right)^{3 / 2} O\left(h^{3 / 2}\right)
\end{aligned}
$$




$$
\begin{aligned}
E\{x(t+h)-\xi \| x(t, \omega) & =\xi\}=\int_{t}^{t+h} m(s, \xi) d s+\left(1+\xi^{2}\right)^{1 / 2} O\left(h^{3 / 2}\right), \\
E\left\{[x(t+h)-\xi]^{2} \| x(t, \omega)\right. & =\xi\}=\int_{t}^{t+h} \sigma^{2}(s, \xi) d s+\left(1+\xi^{2}\right) O\left(h^{3 / 2}\right) .
\end{aligned}
$$

Here each $O$ term is uniform in the variable $\xi$, and also in $t$ for $t$ in a compact set. If the diffusion coefficients do not depend on the parameter, so that we are in the stationary case, the integrals on the right can be evaluated, and the $O$ terms on the right will of course be uniform in $t$. The evaluations make clear the significance of the diffusion coefficients as the differential increments of mean and variance.

Conversely, the author has proved [2, p. 287] that, under appropriate conditions, including evaluations slightly weaker than (2.3), (2.4), and (2.5), a Markov process will necessarily be a standard Ito process.

In the following, we shall frequently consider the process $\left\{x_{\xi}(t), t \in T\right\}$ for varying initial value $\xi$. When we do this, we shall hold constant the basic measure space on which the specified Brownian motion $y(t)$ process of $(2.1)$ is defined, so that the various $x_{\xi}(t)$ processes will all be defined on the same measure space. We shall need below a measure of the change of $x_{\xi}(t)$ with $\xi$. This will be provided by the following two lemmas.

Lemma 2.1. Let $\phi$ be a Lebesgue measurable function of a real variable $t \in T$, integrable on compact sets. Then, if there are constants $a, b$, with $b \geqq 0$ such that

$$
0 \leqq \phi(t) \leqq a+b \int_{0}^{t} \phi(s) d s, \quad t \in T,
$$

it follows that

$$
\phi(t) \leqq a e^{b t},
$$$$
t \in T \text {. }
$$

To prove the lemma, we observe that, by (2.6),

$$
b^{n+1} \int_{0}^{t} \phi(s) \frac{(t-s)^{n}}{n !} d s \leqq \frac{a t^{n+1} b^{n+1}}{(n+1) !}+b^{n+2} \int_{0}^{t} \phi(s) \frac{(t-s)^{n+1}}{(n+1) !} d s, \quad n \geqq 0 .
$$

Adding (2.6) and these inequalities yields the desired (2.7).

Lemma 2.2. To each compact subinterval $T_{1}$ of $T$ corresponds a constant $c$ such that

$$
E\left\{\operatorname{Max}_{s \leqq t}\left|x_{\xi}(t)-x_{\eta}(t)\right|^{2}\right\} \leqq c|\xi-\eta|^{2}, \quad t \in T_{1} .
$$

Let $K$ be the constant in the conditions SIP imposed on the diffusion coefficients for $t \in T_{1}$. Then we observe that 


$$
\left\{\int_{0}^{s}\left|m\left[r, x_{\xi}(r)\right]-m\left[r, x_{\eta}(r)\right]\right| d r\right\}^{2} \leqq K^{2} t \int_{0}^{t}\left|x_{\xi}(r)-x_{\eta}(r)\right|^{2} d r, \quad s \leqq t .
$$

Next we observe that the integral

$$
I(s)=\int_{0}^{s}\left\{\sigma\left[r, x_{\xi}(r)\right]-\sigma\left[r, x_{\eta}(r)\right]\right\} d y(r)
$$

defines a martingale, as $s$ varies, as does any stochastic integral of this type [2, p. 444] and hence (ibid. pp. 317, 354)

$$
\begin{aligned}
E\left\{\max _{r \leqq s} I(r)^{2}\right\} & \leqq 4 E\left\{I(s)^{2}\right\}=4 E\left\{\int_{0}^{8}\left|\sigma\left[r, x_{\xi}(r)\right]-\sigma\left[r, x_{\eta}(r)\right]\right|^{2} d r\right\} \\
& \leqq 4 K^{2} E\left\{\int_{0}^{t}\left|x_{\xi}(r)-x_{\eta}(r)\right|^{2} d r\right\}, \quad s \leqq t .
\end{aligned}
$$

If we now write (2.1) for $x_{\xi}(t)$ and $x_{\eta}(t)$, and subtract, we find, using the preceding inequalities, and denoting the left side of $(2.8)$ by $M(t)$,

$$
M(t) \leqq 3|\xi-\eta|^{2}+3 K^{2} t \int_{0}^{t} M(r) d r+12 K^{2} \int_{0}^{t} M(r) d r .
$$

Lemma 2.1 can be applied to yield (2.8) with $c$ depending only on the righthand end point of $T_{1}$ and on $K$.

In many cases we shall be able to suppose that the diffusion coefficients are bounded. In this case, the reasoning leading to (2.3), (2.4), and (2.5) can be simplified, and leads to simpler evaluations: the multiplier of the $O$ term on the right, involving $\xi$, is to be omitted in each case. Moreover, in this case, for every real number $\alpha$, if $K_{1}$ is a bound for $m$ and $\sigma$,

$$
E\left\{e^{\alpha x \xi(t)}\right\} \leqq L e^{\alpha \xi}, \quad L=\exp \left(K_{1}+K_{1}|\alpha| t+K_{1} \alpha^{2} t\right) .
$$

To see this, let $K_{1}$ be a bound for $|m|$ and $\sigma$. Then

$$
E\left\{e^{\alpha x \xi(t)}\right\}=E\left\{e^{\alpha \xi+u+v}\right\},
$$

where

$$
|u|=\left|\alpha \int_{0}^{t} m\left[s, x_{\xi}(s)\right] d s\right| \leqq K_{1}|\alpha| t, \quad v=\alpha \int_{0}^{t} \sigma\left[s, x_{\xi}(s)\right] d y(s) .
$$

The inequality (2.9) then follows from the following lemma.

LEMMA 2.3. If $w=\int_{0}^{t} \Phi(s, \omega) d y(s)$, where $|\Phi(s, \omega)| \leqq K_{1}$, then

$$
E\left\{e^{\alpha w}\right\} \leqq e^{K_{1} \alpha^{2} t} \text {. }
$$

Here (see [2, p. 437]) we suppose that $\Phi$ has the usual properties of the integrands in these stochastic integrals. This inequality is easily proved 
using the standard sums approximating these integrals, and the proof will be omitted. Applying (2.9) with $\alpha$ replaced by $-\alpha$, and adding the two inequalities, we deduce

$$
E\left\{e^{\alpha\left|x_{\xi}(t)\right|}\right\} \leqq 2 L e^{\alpha|\xi|}, \quad \alpha>0 .
$$

The following lemma will also be useful below. We restrict our attention to the stationary case, to which the lemma will be applied.

Lemma 2.4. If $\sigma>0$, and if $m, \sigma$ do not depend on $t$, then, for every $t>0$,

$$
\lim _{\epsilon \rightarrow 0} P\left\{\underset{s \leqq \imath}{\operatorname{Max}} x_{\xi}(s, \omega)-\xi \geqq \epsilon\right\}=1,
$$

and this limit is uniform for $\xi$ in any bounded set in which $\sigma$ is bounded from below by a positive constant.

According to [2, pp. 283-284],

$$
x_{\xi}(s)-\xi=\eta_{1}+\eta_{2}+s m(\xi)+\sigma(\xi) y(s),
$$

where

$$
E\left\{\operatorname{Max}_{s \leqq t} \eta_{j}^{2}\right\} \leqq O\left(t^{2}\right)\left(1+\xi^{2}\right), \quad j=1,2,
$$

and the $O$ term does not depend on $\xi$. It is no restriction in proving the asserted limit relation if we allow $t$ to go to 0 with $\epsilon$. If we take $t^{1 / 4}=\epsilon$, and if $\epsilon$ is then so small that $t|m(\xi)| \leqq \epsilon$, we find that

$$
\begin{aligned}
P\left\{\max _{s \leqq t} x_{\xi}(s, \omega)-\xi \geqq \epsilon\right\} \geqq & P\left\{\max _{s \leqq t} y(s, \omega) \geqq 4 \epsilon / \sigma(\xi)\right\} \\
& -\sum_{1}^{2} P\left\{\max _{s \leqq t}\left|\eta_{j}\right| \geqq \epsilon\right\} \\
\geqq & 2 P\left\{y(t, \omega) \geqq 4 t^{1 / 4} / \sigma(\xi)\right\}-2 O\left(t^{2}\right)\left(1+\xi^{2}\right) t^{-1 / 2} .
\end{aligned}
$$

This difference goes to 1 , when $\epsilon \rightarrow 0$, with the required uniformity.

3. Generalization of the standard Ito processes. In this section we shall make a simple generalization of the standard Ito processes, which will be useful in discussing stopped processes. Let $m, \sigma$, and a $y(t)$ process be as in $\S 2$, and let $\mathcal{F}_{t}$ be as defined there. For each $t \in T, i=1,2$, let $\psi_{i}(t)$ be a random variable which is measurable relative to the Borel field $\mathcal{f}_{t}$, with the value $\psi_{i}(t, \omega)$ at the point $\omega$. It is supposed that, if $(t, \omega)$ measure is the direct product of Lebesgue $t$ measure and the given $\omega$ measure, then $\psi_{i}(t, \omega)$ defines a $(t, \omega)$ measurable function, and that

$$
\int_{0}^{t} E\left\{\psi_{i}(s)^{2}\right\} d s<\infty, \quad t \in T .
$$


Consider the equation

$$
x(t)=x(0)+\int_{0^{-}}^{t} m[s, x(s)] \psi_{1}(s) d s+\int_{0}^{t} \sigma[s, x(s)] \psi_{2}(s) d y(s) .
$$

If $\psi_{1}(s)$ and $\psi_{2}(s)$ are, for each value of $s$, constant $\omega$ functions, this equation is only slightly more general than (2.1), because, at least if $\psi_{1}$ and $\psi_{2}$ are bounded functions, $\psi_{1}$ can be absorbed in $m$ and $\psi_{2}$ in $\sigma$. The real point of this generalization is that $\psi_{1}(s)$ and $\psi_{2}(s)$ may be nontrivial $\omega$ functions. In any event, (3.1) can be solved in exactly the same way as (2.1), to yield a stochastic process which has almost all its sample functions continuous, and, with this property, is essentially unique. The process need not necessarily be a Markov process, however. For example, if $m=1, \sigma=0, \psi_{1}(s)=y(s)$, the process will not be a Markov process.

4. Stopped standard Ito processes. Let $\{x(t), 0 \leqq t<\infty\}$ be a standard Ito process. (The changes in the following that would be required by a different choice of parameter interval will be obvious.) We shall now find an equation satisfied by the process after it is stopped at a barrier or stopped in accordance with a criterion of a similar type. Let $\tau$ be a random variable defined on the same measure space as the $x(t)$ process, and satisfying the following conditions.

S1 $0 \leqq \tau(\omega) \leqq \infty$.

S2 For every constant $s,\{\tau(\omega) \leqq s\} \in \mathcal{F}_{s}^{\prime}$, where the Borel field $\mathcal{F}_{8}^{\prime}$ was defined in $\S 2$.

Define

$$
\tilde{x}(t, \omega)=\left\{\begin{array}{lll}
x(t, \omega) & \text { if } & \tau(\omega) \geqq t, \\
x[\tau(\omega), \omega] & \text { if } & \tau(\omega)<t,
\end{array}\right.
$$

that is, the $\tilde{x}(t)$ process is the $x(t)$ process stopped at time $\tau$. If $\tau$ is the first time an $x(t)$ process sample function has a value in some closed set $A$ (or $+\infty$ if there is no such time) the $\tilde{x}(t)$ process is described as the $x(t)$ process stopped at the barrier $A$. Let $\psi(s)$ be the characteristic function of the set $\{\tau(\omega)>s\}$. Then we shall show that, if the $x(t)$ process is a solution of $(2.1)$, the $\bar{x}(t)$ process is given by

$$
\tilde{x}(t)=x(0)+\int_{0}^{t} m[s, x(s)] \psi(s) d s+\int_{0}^{t} \sigma[s, x(s)] \psi(s) d y(s),
$$

where the last integral is defined to make almost all sample functions of the $\tilde{x}(t)$ process continuous. Once this has been shown, it will be possible to replace $x(s)$ in each integrand by $\tilde{x}(s)$ without changing the integrand. In this form, (4.1) becomes an equation of the form (3.1). The $\tilde{x}(t)$ process is thus a generalized Ito process in the sense of $\$ 3$. In the following we shall assume, as we can, that the last integral in (4.1) defines a continuous function of $t$ 
with probability 1 , and shall denote by $x_{1}(t)$ the right side of this equation. If $a \geqq 0$,

$$
x_{1}(t)=x_{1}(a)+\int_{a}^{t} m[s, x(s)] \psi(s) d s+\int_{a}^{t} \sigma[s, x(s)] \psi(s) d y(s) .
$$

The proof of the identification of $x(t)$ with $x_{1}(t)$ will be carried through in two steps.

(i) If $0 \leqq a<t$, then $x_{1}(t)=x_{1}(a)$ almost everywhere on the $\omega$ set $\{\tau(\omega) \leqq a\}$. To prove this it is sufficient to prove that the last term in (4.2) vanishes almost everywhere on this $\omega$ set. Now

$$
\begin{aligned}
\int_{a}^{t} \sigma[s, x(s)] \psi(s) d y(s)= & \int_{a}^{t} \sigma[s, x(s)] \psi(s) \psi(a) d y(s) \\
& +\int_{a}^{t} \sigma[s, x(s)] \psi(s)[1-\psi(a)] d y(s) .
\end{aligned}
$$

Here the last integral vanishes almost everywhere, since the integrand vanishes identically. The first integral on the right can be written in the form

$$
\psi(a) \int_{a}^{t} \sigma[s, x(s)] \psi(s) d y(s) .
$$

(The justification for taking $\psi(a)$ outside the integral sign is trivial from the definition of this integral.) In this form it is obvious that the expression vanishes on $\{\tau(\omega) \leqq a\}$. Thus we have proved the assertion (i), and it follows easily that $x_{1}(t, \omega)=x_{1}[\tau(\omega), \omega]$, when $t \geqq \tau(\omega)$, ignoring an $\omega$ set of probability 0 independent of $t$. The following assertion, when proved, then yields the fact that the $x_{1}(t)$ process and the $x(t)$ process are essentially the same.

(ii) If $t>0, x_{1}(t)=x(t)$ almost everywhere on the $\omega$ set $\{\tau(\omega) \geqq t\}$. To prove this, suppose that

$$
0=t_{0}^{(n)}<\cdots<t_{n}^{(n)}=t
$$

and define

$$
\begin{aligned}
& \psi_{n}(0, \omega)=1, \\
& \psi_{n}(s, \omega)= \begin{cases}1 & \text { if } t_{j}^{(n)}<s \leqq t_{j+1}^{(n)} \text { and } \tau(\omega) \geqq t_{j}^{(n)} \\
0 & \text { otherwise. }\end{cases}
\end{aligned}
$$

Then

$$
\left\{\psi_{n}(s, \omega) \neq \psi(s, \omega)\right\} \subset \bigcup_{j}\left\{t_{j}^{(n)}<\tau(\omega) \leqq t_{j+1}^{(n)}, t_{j}^{(n)}<s \leqq t_{j+1}^{(n)}\right\} .
$$

If we define $(s, \omega)$ measure as the direct product of Lebesgue $s$ measure and 
the given $\omega$ measure, the $(s, \omega)$ measure of the set on the left goes to 0 with $\delta_{n}=\operatorname{Max}_{j}\left(t_{j+1}^{(n)}-t_{j}^{(n)}\right)$ since this measure is at most

$$
\sum_{i}\left(t_{j+1}^{(n)}-t_{j}^{(n)}\right) P\left\{t_{j}^{(n)}<\tau(\omega) \leqq t_{j+1}^{(n)}\right\} \leqq \delta_{n}
$$

Hence

$$
\begin{gathered}
E\left\{\left|\int_{0}^{t}\left[\sigma[s, x(s)] \psi(s)-\sigma[s, x(s)] \psi_{n}(s)\right] d y(s)\right|^{2}\right\} \\
=\int_{0}^{t} E\left\{\left|\sigma[s, x(s)]\left[\psi(s)-\psi_{n}(s)\right]\right|^{2}\right\} d s \rightarrow 0 \text { if } \delta_{n} \rightarrow 0 .
\end{gathered}
$$

We use here the fact that, as follows from the inequality

$$
\sigma^{2}[s, x(s)] \leqq \text { const. }\left[1+x(s)^{2}\right],
$$$$
s \leqq t
$$

the left side of the inequality is $(s, \omega)$ integrable on $[0, t] \times \Omega$. We are now ready to prove assertion (ii). We need only prove that the last term in (4.1) is equal almost everywhere on $\{\tau(\omega) \geqq t\}$ to the same integral without $\psi$ in the integrand. Now if $\delta_{n} \rightarrow 0$, we have, using (4.3),

$$
\begin{aligned}
& \int_{0}^{t} \sigma[s, x(s)] \psi(s) d y(s)=\underset{n \rightarrow \infty}{\lim .} \int_{0}^{t} \sigma[s, x(s)] \psi_{n}(s) d y(s) \\
& =\underset{n \rightarrow \infty}{\lim .} \sum_{1}^{n} \int_{t_{j-1}^{(n)}}^{t_{j}^{(n)}} \sigma[s, x(s)] \psi\left(t_{j-1}^{(n)}\right) d y(s) \\
& =\lim . \sum_{n \rightarrow \infty}^{n} \psi\left(t_{i}^{(n-1)}\right) \int_{t_{j-1}^{(n)}}^{t_{j}^{(n)}} \sigma[s, x(s)] d y(s) .
\end{aligned}
$$

When $\tau(\omega) \geqq t$, this limit becomes

$$
\underset{n \rightarrow \infty}{\lim } \sum_{1}^{n} \int_{t_{j-1}^{(n)}}^{t_{j}^{(n)}} \sigma[s, x(s)] d y(s)=\int_{0}^{t} \sigma[s, x(s)] d y(s),
$$

as was to be proved.

The following theorem is intuitively obvious, and we now have created enough apparatus to make its proof simple.

TheOREM 4.1. Let $\left\{x_{i}(t), t \in T\right\}(i=1,2)$ be a standard Ito process with diffusion coefficients $m_{i}, \sigma_{i}$, and $x_{i}(0)=\xi$. Suppose that the determining Brownian motion process is the same for the two values of $i$, and that there is a finite or infinite closed interval I such that

$$
\begin{aligned}
m_{1}(t, \eta) & =m_{2}(t, \eta), \quad \text { if } \quad t \in T, \eta \in I . \\
\sigma_{1}(t, \eta) & =\sigma_{2}(t, \eta),
\end{aligned}
$$


Suppose that $\xi \in I$, and let $\tau_{i}(\omega)$ be the smallest value of $t$ with $x_{i}(t, \omega)$ an end point of $I$, or $\tau_{i}(\omega)=\infty$ if there is no such value. Then

$$
\begin{aligned}
P\left\{\tau_{1}(\omega)=\tau_{2}(\omega)\right\} & =1, \\
P\left\{x_{1}(t, \omega)=x_{2}(t, \omega) \text { if } t \leqq \tau_{1}(\omega)\right\} & =1 .
\end{aligned}
$$

To prove this theorem, let $\tau=\min \left(\tau_{1}, \tau_{2}\right)$, and let $\left\{\tilde{x}_{i}(t), t \in T\right\}$ be the $x_{i}(t)$ process stopped at time $\tau$. If $\psi(s)$ is the characteristic function of the $\omega$ set $\{\tau(\omega) \geqq s\}$, we have, from (4.1), and using the hypotheses on the diffusion coefficients,

$$
\tilde{x}_{i}(t)=\xi+\int_{0}^{t} m_{1}\left[s, \tilde{x}_{i}(s)\right] \psi(s) d s+\int_{0}^{t} \sigma_{1}\left[s, \tilde{x}_{i}(s)\right] \psi(s) d y(s), \quad i=1,2 .
$$

These equations are of the form (3.1). Hence there is essentially only one solution with continuous sample functions, and this fact implies the truth of the theorem.

We now compute the infinitesimal properties of a stopped standard Ito process. We assume for definiteness in the following that the parameter set is a finite closed interval $T$. If $t$ is a point of this interval, we set $T_{t}=T[t, \infty]$. We work in the following with a specified pair of diffusion coefficients, with parameter interval $T$, and a specified Brownian motion process which we shall use to define various standard Ito processes. Let $\left(r_{1}, r_{2}\right)$ be a finite or semiinfinite interval, let $\xi$ be a point of this interval, let $\left\{x(s), s \in T_{t}\right\}$ be a standard Ito process with $x(t)=\xi$, and let $\left\{\tilde{x}(s), s \in T_{t}\right\}$ be this process stopped when its trajectories reach $r_{1}$ or $r_{2}$, if ever. Then

$$
P\left\{\max _{t \leqq u \leqq t+h}|\tilde{x}(u, \omega)-\xi| \geqq \epsilon\right\}=\left(1+\xi^{2}\right)^{3 / 2} O\left(h^{3 / 2}\right),
$$

where $O\left(h^{3 / 2}\right)$ is uniform in the pair $(t, \xi)$. This evaluation is simply a translation of the corresponding evaluation (2.3) for the unstopped process. Let $\tau$ be the stopping time, and let $\psi(s)$ be the characteristic function of the $\omega$ set $\{\tau(\omega) \geqq s\}$. Then we write

$$
\begin{aligned}
\bar{x}(t+h)-\xi= & \int_{t}^{t+h}[m[s, x(s)]-m(s, \xi)] \psi(s) d s \\
& +\int_{t}^{t+h}[\sigma[s, x(s)]-\sigma(s, \xi)] \psi(s) d y(s) \\
& +\int_{t}^{t+h} m(s, \xi) \psi(s) d s+\int_{t}^{t+h} \sigma(s, \xi) \psi(s) d y(s) \\
& =\mathrm{I}+\mathrm{II}+\mathrm{III}+\mathrm{IV} .
\end{aligned}
$$

Now it is easily calculated (see $[2$, p. $284(3.16)]$ ) that 


$$
E\left\{I^{2}\right\} \leqq E\left\{\max _{\imath \leqq u \leqq t+h}\left|\int_{t}^{u}[m[s, x(s)]-m(s, \xi)] d s\right|^{2}\right\} \leqq L_{1}\left(1+\xi^{2}\right) h^{3},
$$

for some constant $L_{1}$. Moreover, if $\mathrm{II}^{\prime}$ is the same as II except that the factor $\psi(s)$ is omitted from the integrand, it is easily calculated (see $[2$, p. 284, (3.17) ]) that

$$
E\left\{\mathrm{II}^{2}\right\} \leqq E\left\{\mathrm{II}^{\prime 2}\right\} \leqq L_{2} h^{2}\left(1+\xi^{2}\right),
$$

for some constant $L_{2}$. We now deduce

$$
\begin{aligned}
\text { (4.5) } E\{\tilde{x}(t+h)-\xi\} & =\int_{t}^{t+h} m(s, \xi) E\{\psi(s) d s\}+\left(1+\xi^{2}\right)^{1 / 2} O\left(h^{3 / 2}\right), \\
\text { (4.6) } E\left\{[\tilde{x}(t+h)-\xi]^{2}\right\} & =\int_{t}^{t+h} \sigma(s, \xi)^{2} E\{\psi(s)\} d s+\left(1+\xi^{2}\right) O\left(h^{3 / 2}\right),
\end{aligned}
$$

where $O\left(h^{3 / 2}\right)$ is uniform in the pair $(t, \xi)$. Here $E\{\psi(s)\}=P\{\tau(\omega) \geqq s\}$. To put these equations in more usable form, note that, if $\delta$ is sufficiently small,

$$
\begin{aligned}
P\{\psi(t+h, \omega)=1\} & =P\{\tau(\omega) \geqq t+h\} \\
& \geqq P\left\{\max _{t \leqq u \leqq t+h}|x(u)-\xi| \leqq \delta\right\} \\
& =1-\left(1+\xi^{2}\right)^{3 / 2} O\left(h^{3 / 2}\right),
\end{aligned}
$$

where $O\left(h^{3 / 2}\right)$ is uniform as above. Hence, for $\xi$ at distance greater than $\delta$ from $r_{1}, r_{2}$,

$$
E\{\tilde{x}(t+h)-\xi\}=\int_{t}^{t+h} m(s, \xi) d s+\left(1+\xi^{2}\right)^{3 / 2} O\left(h^{3 / 2}\right),
$$

where $O\left(h^{3 / 2}\right)$ is uniform as above. Similarly, for $\xi$ at distance greater than $\delta$ from $r_{1}, r_{2}$,

$$
E\left\{[\bar{x}(t+h)-\xi]^{2}\right\}=\int_{t}^{t+h} \sigma(s, \xi)^{2} d s+\left(1+\xi^{2}\right)^{3 / 2} O\left(h^{3 / 2}\right) .
$$

Let $\epsilon$ be any positive number, and define the usual truncated differential mean and variance,

$$
\begin{aligned}
& \int_{\xi-\epsilon}^{\xi+\epsilon}(\eta-\xi) d_{\eta} P\{x(t+h, \omega) \leqq \eta\}, \\
& \int_{\xi-\epsilon}^{\xi+\epsilon}(\eta-\xi)^{2} d_{\eta} P\{x(t+h, \omega) \leqq \eta\} .
\end{aligned}
$$

According to (2.3), these quantities differ from the left sides of (4.8) and 
(4.9) respectively by $\left(1+\xi^{2}\right)^{3 / 2} O\left(h^{3 / 2}\right)$, and thus these quantities, the corresponding left sides of (4.8), (4.9) and (2.4), (2.5) are interchangeable up to errors of the above order of magnitude. Although the left sides of (2.4) and (2.5) have the clearest intuitive significance, the truncated means in (4.10) and (4.11) have certain advantages, and have been used by Feller [4] in his proofs of existence theorems for diffusion processes.

According to our comparison of (2.5) and (4.11),

$$
\int_{|\eta-\xi|>\epsilon}(\eta-\xi)^{2} d_{\eta} P\{x(t+h, \omega) \leqq \eta\}=\left(1+\xi^{2}\right) O\left(h^{3 / 2}\right),
$$

where $O\left(h^{3 / 2}\right)$ is, as usual, uniform in the pair $(t, \xi)$. This makes specific the Lindeberg condition which makes the right side $o(h)$.

Finally, we remark that, if $r_{1}$ and $r_{2}$ are finite, then the power of $\left(1+\xi^{2}\right)$ which appears in the inequalities involving the stopped process is of course unnecessary.

5. Ito processes with general state intervals. In this section we shall generalize the standard Ito processes by supposing that the diffusion coefficients are defined only for the state variable $\xi$ in some interval $I$ which we shall call the state interval. The (Markov) processes we obtain are the subject of the present paper. The interval $I$ may be closed, open, or neither, finite or infinite, but if infinite it will contain only finite points. We denote its end points by $r_{1}, r_{2}, r_{1}<r_{2}$. The hypotheses on the diffusion coefficients are that they be Baire functions of $(t, \xi)$ in $T \times I$, and that, if $t$ is restricted to a compact subset of $T$, and $\xi$ to a compact subset of $I$, there be a corresponding constant $K$, depending on the specified compact sets, such that

$$
|m(t, \xi)| \leqq K, \quad 0 \leqq \sigma(t, \xi) \leqq K,
$$

IP

$$
\left|m\left(t, \xi_{2}\right)-m\left(t, \xi_{1}\right)\right| \leqq K\left|\xi_{2}-\xi_{1}\right|, \quad\left|\sigma\left(t, \xi_{2}\right)-\sigma\left(t, \xi_{1}\right)\right| \leqq K\left|\xi_{2}-\xi_{1}\right| .
$$

The standard Ito case of $\S 2$ is thus a special case in which $I$ is the interval $(-\infty, \infty)$ and in which there is a special uniformity condition imposed on the above inequalities as the compact set on which they are to hold varies.

Let $T$ be any interval with left-hand end point 0 , let $\{y(t), 0 \leqq t<\infty\}$ be a Brownian motion process, and let $x(0)$ be a random variable defined on the same measure space as the $y(t)$ process, but independent of this process. We shall now define an Ito process with parameter set $T$ and state interval $I$, in terms of the specified diffusion coefficients satisfying IP, initial value, and Brownian motion process.

First suppose that $I$ is compact. Then the diffusion coefficients can be defined for $t \in T$ and all $\xi$, with the old values in $T \times I$, and satisfying the hypotheses SIP of $\$ 2$ imposed on the diffusion coefficients of a standard Ito process. Let $\left\{x_{1}(t), t \in T\right\}$ be the standard Ito process determined by the 
extended diffusion coefficients, the specified Brownian motion process, and specified $x_{1}(0)=x(0)$. Let $\{x(t), t \in T\}$ be this process stopped at the end points of $I$. The $x(t)$ process will be called an Ito process with state interval $I$. The fact that it is essentially independent of the way the diffusion coefficients are extended is a consequence of Theorem 4.1. In particular, if the original diffusion coefficients are functions of $\xi$ alone, their extensions can be made to have this property also. Hence it is clear that in this case the $x(t)$ process will have stationary transition probabilities.

If the state interval is not compact, suppose next that its left-hand end point $r_{1}$ is finite and is in the interval. Let $\left\{I_{n}, n \geqq 1\right\}$ be a monotone increasing sequence of compact intervals whose union is $I$, with common lefthand end point $r_{1}$. Let $s_{n}$ be the right-hand end point of $I_{n}$, and define $x_{n}(0)$ by

$$
x_{n}(0)=\min \left[x(0), s_{n}\right] \text {. }
$$

Define $\left\{x_{n}(t), t \in T\right\}$ as the Ito process with the state interval $I_{n}$, specified diffusion coefficients, initial position $x_{n}(0)$, and specified Brownian motion process. For $m<n$ the $x_{m}(t)$ process is nearly the $x_{n}(t)$ process stopped at $s_{m}$, and in fact we can suppose that

$$
x_{m}(t, \omega)=x_{n}(t, \omega) \quad \text { if } \quad x_{n}(t, \omega) \in I_{m}, \quad s \leqq t .
$$

Then $\lim _{n \rightarrow \infty} x_{n}(t, \omega)$ exists for each $\omega$, and defines the desired process $\{x(t), t \in T\}$. It is not a priori obvious that the sample functions of this process are almost all continuous. In fact, it is conceivable that, if $x(t, \omega)=r_{2}$, the function $x(\cdot, \omega)$ oscillates back and forth between points arbitrarily near some inner point of $I$ which we shall call the left oscillation limit and points arbitrarily near $r_{2}$, for the argument in the neighborhood of a point $\tau(\omega) \leqq t$, so that $\tau(\omega)$ is an oscillatory discontinuity of $x(\cdot, \omega)$ on the left, with $x(s, \omega)$ $=r_{2}$ for $s \geqq \tau(\omega)$. We shall call such a point $\omega$ an oscillatory point. To prove that almost all sample functions of the $x(t)$ process are continuous, we prove that the class of oscillatory points with left oscillation limit $\leqq \alpha$ has probability 0 , for every $\alpha \in I$. Let $\xi_{1}$ and $\xi_{2}$ be points of $I$, with $\alpha<\xi_{1}<\xi_{2}$, and let $\tau_{n}^{(0)}(\omega)$ be the time $x_{n}(\cdot, \omega)$ first has the value $\xi_{1}$, let $\tau_{n}^{(0)}(\omega)+\tau_{n}^{(1)}(\omega)$ be the time $x_{n}(\cdot, \omega)$ first has the value $\xi_{1}$ after having already had the values $\xi_{1}$ and $\xi_{2}$ in that order, and in general let $\tau_{n}^{(k)}(\omega)$ for $k>0$ be the time between the $k$ th and $(k+1)$ th times $x(\cdot, \omega)$ has the value $\xi_{1}$, with $\xi_{2}$ assumed in between. If $\omega$ is oscillatory, with left hand oscillation limit $\leqq \alpha$, and if $k$ is arbitrary, $\tau_{n}^{(k)}(\omega)$ is defined for $n$ sufficiently large, so that $\tau^{(k)}(\omega)=\lim _{n \rightarrow \infty} \tau_{n}^{(k)}(\omega)$ is well defined. Now, by (4.4), there is a constant $L$ such that

$$
P\left\{\max _{0 \leqq h \leqq \delta}|x(t+h)-\xi| \geqq \epsilon|| x_{n}(t, \omega)=\xi\right\} \leqq L \delta^{3 / 2}
$$

for $t, t+h$ in a compact subset of $T$, and $\xi_{1} \leqq \xi \leqq \xi_{2}$. It follows that, if $\epsilon=\xi_{2}-\xi_{1}$, 


$$
P\left\{\tau^{(k)}(\omega) \leqq \delta \| \tau^{(0)}, \cdots, \tau^{(k)}\right\} \leqq L \delta^{3 / 2} .
$$

(If the reader is disturbed by the fact that the random variables here are not everywhere defined, he may define them as -1 where they are not already defined. The above evaluation is then of course only to be valid where the conditioning variables are non-negative.) This evaluation makes it impossible that $\sum_{k} \tau^{(k)}(\omega)$ converge with positive probability, whereas from the definition this series has sum $\leqq t$ on the set of oscillatory points with left oscillation limit $\leqq \alpha$. Hence there are almost no such oscillatory points, and therefore almost no oscillatory points whatever. The $x(t)$ process we have defined thus has almost no discontinuous sample functions. It is essentially determined by the diffusion coefficients, $x(0)$, and the specified Brownian motion process. It has a transition probability function uniquely determined by the diffusion coefficients.

If the state interval $I$ has a finite right-hand end point, which it contains, but is open on the left, Ito processes with state interval $I$ are defined similarly. Finally, if $I$ is open, the only change necessary in the above argument is that $\left\{I_{n}, n \geqq 1\right\}$ is now a monotone sequence of compact subsets of $I$, with union $I$.

For any state interval $I$, if an Ito process $\{x(t), t \in T\}$ with this state interval is stopped when its sample functions meet the end points of a subinterval $I_{1}$, and if $x(0, \omega) \in I_{1}$, then the stopped process is an Ito process with the state interval $I_{1}$.

If an Ito process has state interval $I$, and if $I_{0}$ is the open kernel of $I$, essentially the same process is obtained if $I_{0}$ is used as state interval.

If $I$ is not finite, our definition may allow the random variables of an Ito process with this state interval to be infinite valued with positive probability, but this will cause no difficulty below.

6. Semigroup generated by an Ito process. From now on we shall deal only with diffusion coefficients which are functions of the state variable only, so that the corresponding Ito processes will have stationary transition probabilities, and the parameter interval will be $[0, \infty)$. This fact will not be mentioned explicitly below. Under this added hypothesis, the conditions IP of $\S 5$ imposed on the diffusion coefficients become the following. The two coefficients are Baire functions defined on the state interval $I$. To every compact subset $I_{0}$ of $I$ corresponds a constant $K$ such that

$$
0 \leqq \sigma(\xi) \text { if } \xi \in I,
$$

IPs

$$
\begin{array}{r}
\left|m\left(\xi_{2}\right)-m\left(\xi_{1}\right)\right| \leqq K\left|\xi_{2}-\xi_{1}\right|, \quad\left|\sigma\left(\xi_{2}\right)-\sigma\left(\xi_{1}\right)\right| \leqq K\left|\xi_{2}-\xi_{1}\right| \\
\text { if } \xi_{j} \in I_{0} .
\end{array}
$$

In the standard Ito case of $\S 2$, the state interval is $(-\infty, \infty)$, but these inequalities are nevertheless to hold throughout $I$, with a single constant $K$. Let $\{x(t), 0 \leqq t<\infty\}$ be an Ito process with state interval $I$. If there is a 
preassigned constant initial value $\xi$, we shall write $x_{\xi}(t)$ for $x(t)$, as usual. Define $U_{t} f$ by

$$
\left(U_{t} f\right)(\xi)=E\left\{x_{\xi}(t)\right\}=E\{f[x(t)] \| x(0, \omega)=\xi\},
$$

as in $\$ 1$. We would like to define a Banach space of functions $f$ with the property that $U_{t}$ takes the Banach space into itself, and that the semigroup is strongly continuous. The infinitesimal operator of the semigroup would then be expected to become the differential operator $D$ defined by (1.5), at least for the functions of interest. It turns out to be simpler, and adequate to our purposes, to renounce the use of general semigroup theorems, and apply Theorem 1.1 in their place. Furthermore we shall reduce problems involving general state intervals to problems involving standard Ito processes in which we shall be able to prescribe the character of the diffusion coefficients outside a finite interval. For some purposes, the simplest choice would be to make the diffusion coefficients vanish identically outside a finite interval, but this choice would not be appropriate for all our applications.

In view of these and other considerations, we make the following choices. We shall assume throughout that the process in question is a standard Ito process with bounded diffusion coefficients. We shall use as the domain of the $U_{t}$ semigroup the Banach space $C$ of functions defined and continuous on $(-\infty, \infty)$, with

$$
\|f\|=\sup _{\xi}|f(\xi)| e^{-|\xi|}<\infty .
$$

With these assumptions, we find, using (2.10),

$$
\left|\left(U_{t} f\right)(\xi)\right| \leqq 2\|f\| L e^{|\xi|},
$$

where $L$ is specified in (2.9). To prove that $U_{t} f$ is continuous, from which it will follow that $U_{t}$ carries $C$ into $C$, we remark first that, from Lemma 2.2, $x_{\eta}(t) \rightarrow x_{\xi}(t)$ in probability when $\eta \rightarrow \xi$, for each value of $t$, so that $f\left[x_{\eta}(t)\right]$ $\rightarrow f\left[x_{\xi}(t)\right]$ in probabiity also. Thus to prove continuity of $U_{t} f$, that is, to prove that

$$
E\left\{f\left[x_{\eta}(t)\right]\right\} \rightarrow E\left\{f\left[x_{\xi}(t)\right]\right\},
$$

we need only justify taking the limit under the expectation symbol, and this justification is provided by the fact that, using (2.10),

$$
E\left\{f\left[x_{\eta}(t)\right]^{2}\right\} \leqq\|f\|^{2} E\left\{e^{2\left|x_{\eta}(t)\right|}\right\} \leqq\|f\|^{2} L e^{2|\eta|},
$$

where $L$ (not equal to the $L$ in (6.1)) is specified in (2.9), and does not depend on $\eta$, so that the left side of (6.2) is bounded independently of $\eta$ in a neighborhood of $\xi$. We have now proved that $U_{t}$ takes $C$ into $C$. The operator $U_{t}$ is linear, and has norm $\leqq 2 L$, according to (6.1). Here $L$ depends on $t$ but is bounded on every compact $t$ set. It is not difficult to prove that, if $C_{1}$ is the subclass of $C$ whose functions are absolutely continuous, with 


$$
\sup _{\xi}\left|f^{\prime}(\xi)\right| e^{-|\xi|}<\infty,
$$

then $U_{t}$ takes $C_{1}$ into $C_{1}$, and $U_{t} f$, for $f \in C_{1}$, defines a strongly continuous function of $t$ on $[0, \infty)$. Since we shall not need this fact, we omit its proof. What we shall need is the now trivial fact that the semigroup is of type $\Gamma$ as defined in $\$ 1$.

7. The infinitesimal operator of a standard Ito process. Let $\{x(t)$, $0 \leqq t<\infty\}$ be a standard Ito process with bounded diffusion coefficients. Define the differential operator $D$ as usual by

$$
D=\frac{\sigma^{2}}{2} \frac{d^{2}}{d \xi^{2}}+m \frac{d}{d \xi} .
$$

Suppose that $f$ is a function defined on $(-\infty, \infty)$, with two continuous derivatives, and that outside some finite interval $f$ has a third continuous derivative with

$$
\sup _{\xi} e^{-|\xi|}\left|f^{\prime \prime \prime}(\xi)\right|<\infty .
$$

The class of such functions is a linear, but not closed, subspace of $C$, which we shall denote by $C_{2}$. If $f \in C_{2}$, and if $\epsilon>0$, we have, using the notation of $\S 1$,

$$
\begin{aligned}
\frac{1}{h}\left(U_{h} f-f\right)(\xi)= & \frac{1}{h} \int_{\xi-\epsilon}^{\xi+\epsilon}\left[(\eta-\xi) f^{\prime}(\xi)\right. \\
& \left.+\frac{(\eta-\xi)^{2}}{2} f^{\prime \prime}(\xi)+(\eta-\xi)^{2} \alpha(\xi, \eta)\right] p(h, \xi, d \eta)+\beta \\
= & D f+O\left(h^{1 / 2}\right)+\frac{1}{h} \int_{\xi-\epsilon}^{\xi+\epsilon}(\eta-\xi)^{2} \alpha(\xi, \eta) p(h, \xi, d \eta)+\beta
\end{aligned}
$$

where

$$
\beta=\frac{1}{h} \int_{|\eta-\xi|>\epsilon}[f(\eta)-f(\xi)] p(h, \xi, d \eta) .
$$

Here we have used the evaluations found in $\$ 4$ for the truncated expectations (4.10) and (4.11). The $O\left(h^{1 / 2}\right)$ is uniform in the variable $\xi$ on $(-\infty, \infty)$. We shall show that the left side of (7.2) converges strongly to $D f$ in the sense of Banach space $C$ distance. We need only show that the last two terms on the right in (7.2) converge strongly to 0 . Taking the last term first, we note that

$$
e^{-|\xi|}|\beta| \leqq \frac{e^{-|\xi|}}{h} \int_{|\eta-\xi|>\epsilon}|f(\eta)| p(h, \xi, d \eta)+\frac{\|f\|}{h} P\left\{\left|x_{\xi}(h, \omega)-\xi\right| \geqq \epsilon\right\} .
$$

The second term on the right goes to 0 uniformly on $(-\infty, \infty)$, according to (2.3), since, as we remarked in $\S 2$, the factor involving $\xi$ on the right in (2.3) 
is absent if the diffusion coefficients are bounded. The first term on the right in (7.3) is dominated, according to Holder's inequality, by

$$
\begin{aligned}
\frac{e^{-|\xi|}}{h} P^{3 / 4}\left\{\left|x_{\xi}(h, \omega)-\xi\right|\right. & \geqq \epsilon\} E^{1 / 4}\left\{\left|f\left[x_{\xi}(t)\right]\right| 4\right\} \\
& \leqq \frac{\|f\|}{h} L^{1 / 4} P^{3 / 4}\left\{\left|x_{\xi}(h, \omega)-\xi\right|>\epsilon\right\}=\|f\| L^{1 / 4} O\left(h^{1 / 8}\right) .
\end{aligned}
$$

Here $L$ is evaluated in (2.9), and $O\left(h^{1 / 8}\right)$ is uniform on $(-\infty, \infty)$. Thus $\beta$ in (7.2) goes strongly to 0 when $h \rightarrow 0$. There remains the proof that

$$
\lim _{h \rightarrow 0} \frac{e^{-|\xi|}}{h} \int_{\xi-\epsilon}^{\xi+\epsilon}(\eta-\xi)^{2} \alpha(\xi, \eta) p(h, \xi, d \eta)=0
$$

uniformly on $(-\infty, \infty)$. According to (4.12), in which we can ignore the factor $\left(1+\xi^{2}\right)$ since the diffusion coefficients are bounded in the present case, we can choose $\epsilon$ as small as desired in proving (7.4). Since $f$ has two continuous derivatives, $\alpha(\xi, \eta)$ can be made uniformly small for $\xi$ in any finite interval by choosing $\epsilon$ small. If $\epsilon_{1}>0$, and if $\epsilon$ is chosen so small that $|\alpha(\xi, \eta)| \leqq \epsilon_{1}$ in (7.4), the expression after the limit symbol is dominated by

$$
\frac{\epsilon_{1} e^{-|\xi|}}{h} \int_{\xi-\epsilon}^{\xi+\epsilon}(\eta-\xi)^{2} p(h, \xi, d \eta)=\epsilon_{1} e^{-|\xi|}\left[\frac{\sigma(\xi)^{2}}{2}+O\left(h^{1 / 2}\right)\right],
$$

where $O\left(h^{1 / 2}\right)$ is uniform in $\xi$. Since the right side of (7.5) can be made arbitrarily and uniformly small in $(-\infty, \infty)$ by proper choice of $\epsilon_{1}$, that is, by proper choice of $\epsilon$, the limit in (7.4) is 0 pointwise, and uniformly on every finite $\xi$ interval. To prove that the limit is also a strong limit, we must discuss the approach to the limit for large $\xi$. Let $a$ be a bound for $\left|f^{\prime \prime \prime}(\xi)\right| e^{-|\xi|}$ outside some finite interval. Then, for $\xi$ sufficiently large, the expression after the limit symbol in (7.4) is dominated by

$$
\begin{aligned}
\frac{a}{6 h} \int_{\xi-\epsilon}^{\xi+\epsilon}|\eta-\xi|^{3} p(h, \xi, d \eta) & \leqq \frac{a \epsilon}{6 h} \int_{\xi-\epsilon}^{\xi+\epsilon}(\eta-\xi)^{2} p(h, \xi, d \eta) \\
& \leqq \frac{a \epsilon}{6}\left[\frac{\sigma(\xi)^{2}}{2}+O\left(h^{1 / 2}\right)\right],
\end{aligned}
$$

where $O\left(h^{1 / 2}\right)$ is uniform for $\xi \in(-\infty, \infty)$, and this expression is uniformly small if $\epsilon$ is small. Thus we have now proved that

$$
\text { strong } \lim \frac{U_{h} f-f}{h}=D f, \quad f \in C_{2} \text {, }
$$

that is, we have shown that the infinitesimal operator of the $U_{t}$ semigroup is defined on $C_{2}$ and coincides with $D$ there. 
8. The basic martingale theorems for Ito processes. The following treatment of a classical problem is easily made precise. Frankie and Johnny play a game repeatedly. In each game, Frankie either wins or loses a dollar, with probability $1 / 2$ for each. The games are independent, and the problem is to find the probability $p$ of Frankie's eventual ruin if she has initial fortune $a$ and Johnny has initial fortune $b$. Since the game is fair, Frankie's final expected fortune should be her initial fortune $a$, and on the other hand it is calculated at once to be

$$
p \cdot 0+(1-p)(a+b) .
$$

Thus we obtain the classical solution

$$
p=\frac{b}{a+b} .
$$

This method is precisely the method to be used in this paper to find the probability that an Ito process trajectory will reach a specified boundary point of its state interval. It depends on an analysis of the concept of fairness of a game in terms of expectations, yielding the theorem that a martingale goes into a martingale under optimal stopping.

The following theorem is the basis for the application of martingale theory to Ito processes.

Theorem 8.1. Let $\{x(t), 0 \leqq t<\infty\}$ be a standard Ito process with bounded diffusion coefficients and $\sigma>0$. Suppose that

$$
E\left\{e^{|x(0)|}\right\}<\infty \text {. }
$$

If $g$ is in class $C_{2}$ and satisfies

$$
D g=0
$$

or

$$
D g=\lambda g
$$

or

$$
D g=\lambda,
$$

then the corresponding stochastic process

$$
\{g[x(t)], 0 \leqq t<\infty\}
$$

or

$$
\left\{e^{-\lambda t} g[x(t)], 0 \leqq t<\infty\right\}
$$




$$
\{g[x(t)]-\lambda t, 0 \leqq t<\infty\}
$$

is a martingale. If " $=$ " is replaced in any one of the equations (8.3) by " $\geqq$ ", the corresponding stochastic process is a semimartingale, if its random variables have finite expectations.

To prove this theorem, we need only remark that, if the semigroup $\left\{U_{t}, t \geqq 0\right\}$ is defined as in $\S 6$, on the Banach space $C$, it is of type $\Gamma$ in the sense of $\$ 1$. Then Theorem 1.1 is applicable, and its application is feasible here because we have identified the infinitesimal operator of the semigroup with the differential operator $D$ on $C_{2}$. The theorem then follows in accordance with the argument detailed in $\$ 1$, if we can show, in the martingale case, that the random variables of the stochastic processes in question have finite expectations. This fact follows from (2.10) in view of the inequality (in which we assume that $g \in C$ )

$$
E\{|g[x(t)]|\} \leqq\|g\| E\left\{e^{|x(t)|}\right\} \leqq 2 L E\left\{e^{|x(0)|}\right\} .
$$

The right side of this inequality is finite by hypothesis.

We have made no attempt to minimize the hypotheses of this theorem, because the main emphasis of the paper is on the following theorem, which is deduced from Theorem 8.1 but whose hypotheses are nearer the minimal ones because the state interval is compact. However, as a simple example of the direction in which Theorem 8.1 can be improved with little effort, we remark that, if $\alpha>0$, the norming function $e^{|\xi|}$, which is used in the definition of the classes $C$ and $C_{2}$, and in condition (8.2), can be replaced by $e^{\alpha|\xi|}$ with no change in any of the previous discussion or present proof except for the insertion of $\alpha$ in the obvious places. If $\alpha<1$ we thus obtain a slight extension of Theorem 8.1.

As an example of the application of Theorem 8.1, let $x(0)=0, m=0$, $\sigma=1$, so that the $x(t)$ process is a Brownian motion process. The fact that this process is itself a martingale is a (trivial) fact which is asserted in case (a) of the theorem. If $\lambda=1$ and -1 in case (b), we find that the stochastic processes

$$
\left\{e^{21 / 2 x(t)-t}, 0 \leqq t<\infty\right\}, \quad\left\{e^{t} \cos 2^{1 / 2} x(t), 0 \leqq t<\infty\right\}
$$

are martingales. Finally, if $\lambda=1$ in case (c), we find that the stochastic process

$$
\left\{x(t)^{2}-t, 0 \leqq t<\infty\right\}
$$

is a martingale. These assertions are readily proved directly. As an example of the application of case (a) with inequality, we apply it to the above $x(t)$ process, and conclude that if $\phi$ is a convex function with two continuous derivatives, the process $\{\phi[x(t)], 0 \leqq t<\infty\}$ is a semimartingale if its random variables have finite expectations. This follows from general martingale theory even if $\phi$ is only supposed convex and continuous, but not necessarily having derivatives, since the $x(t)$ process is itself a martingale. 
TheOREM 8.2. Let $\{x(t), 0 \leqq t<\infty\}$ be an Ito process with a compact state interval $I$ on which $\sigma>0$. Let $\tau$ be the time of first meeting of a trajectory with the boundary of $I$, and define $\tau_{t}=\min [\tau, t]$. Then if $g$ is continuous on $I$, with two continuous derivatives there, and if it satisfies one of the equations (8.3), the corresponding stochastic process

$$
\{g[x(t)], 0 \leqq t<\infty\}
$$

or

$$
\left\{e^{-\lambda \tau_{\imath} g}[x(t)], 0 \leqq t<\infty\right\}
$$

or

$$
\left\{g[x(t)]-\lambda \tau_{t}, 0 \leqq t<\infty\right\}
$$

is a martingale. If " =" is replaced in any one of the equations (8.3) by " $\geqq$ ", the corresponding stochastic process is a semimartingale.

It is to be understood that $\tau(\omega)=\infty$ if $\omega$ yields an $x(t)$ process trajectory which never reaches the boundary of $I$.

We prove the theorem by reducing it to Theorem 8.1. There is a pair $m_{1}, \sigma_{1}$ of bounded diffusion coefficients, not depending on $t$, with $\sigma_{1}$ always positive, satisfying the conditions imposed on the diffusion coefficients of standard Ito processes, coinciding on $I$ with the given pair $m, \sigma$, and satisfying the equations

$$
m_{1}(\xi)=0, \quad \sigma_{1}(\xi)^{2}=2|\lambda|
$$

for sufficiently large $|\xi|$. Define $D_{1}$ by

$$
D_{1}=\frac{\sigma_{1}^{2}}{2} \frac{d^{2}}{d \xi^{2}}+m_{1} \frac{d}{d \xi} .
$$

Let $\left\{x_{1}(t), 0 \leqq t<\infty\right\}$ be a standard Ito process determined by this pair of diffusion coefficients, the initial condition $x_{1}(0)=x(0)$, and the Brownian motion process used in specifying the $x(t)$ process. The latter process is then the $x_{1}(t)$ process stopped at the boundary of $I$. Let $g_{1}$ satisfy (8.3a), or (8.3b), or (8.3c) with $D$ replaced by $D_{1}$, and be identical with the given function $g$ satisfying the corresponding equation $(8.3 \mathrm{a})$ or $(8.3 \mathrm{~b})$ or $(8.3 \mathrm{c})$ on $I$. Then in case (a), $g_{1}$ is linear outside some finite interval; in case (b), $g_{1}$ is either a linear combination of $e^{\xi}, e^{-\xi}$ or of $\sin \xi$, $\cos \xi$ outside some finite interval, depending on the sign of $\lambda$; and in case (c), $g_{1}$ is a polynomial of degree $\leqq 2$ outside some finite interval. In all cases $g_{1} \in C_{2}$. Hence Theorem 8.1 is applicable to the $x_{1}(t)$ process, and we find that the process $(8.4 \mathrm{a}),(8.4 \mathrm{~b})$, or $(8.4 \mathrm{c})$ as the case may be, with $g$ replaced by $g_{1}$, is a martingale. Hence, according to a standard martingale theorem, the process stopped at the boundary of $I$ is also a martingale. The stopped process is precisely the corresponding process (8.5) 
(a) or (b) or (c). If " $=$ " is replaced in one of the equations (8.3) by " $\geqq$, we denote the resulting inequality by $\left(8.3^{\prime}\right)(\mathrm{a})$ or $(\mathrm{b})$ or $(\mathrm{c})$ as the case may be. If $g$ satisfies one of these inequalities on $I$, define $m_{1}$ and $\sigma_{1}$ as above, and define $g_{1}$ on the line, to be identical with $g$ on $I$, to satisfy this inequality (with $D, g$ replaced by $D_{1}, g_{1}$ ) on the line, and to satisfy the corresponding equality outside some finite interval. This can be done in case (b), for example, as follows. Let $\Psi$ be a function defined on $(-\infty, \infty)$ with continuous second derivatives, nonnegative, zero outside some finite interval, and with $\Psi=D g$ in $I$. Then we can take $g_{1}$ as that solution of $D_{1} f=\Psi$ which coincides with $g$ in $I$. The argument which proved the theorem in the martingale case now extends to the present case.

Since the diffusion coefficients of an Ito process are defined only in the state interval, a function $g$ satisfying any one of the equations (8.3) is defined only in the state interval. On the other hand, the sample functions of an Ito process with a noncompact state interval may take on with positive probability a value which is an end point of the state interval even though this end point is not in the interval. Thus the statement of Theorem 8.2 cannot apply to Ito processes with noncompact state intervals without further restrictions or conventions, because $g[x(t, \omega)]$ will not be defined if $x(t, \omega)$ is an end point of the state interval which is not in the interval. It is therefore somewhat unexpected that we can generalize Theorem 8.2 as simply as in the following theorem. The natural conventions to be made are explained after the statement of the theorem.

ThEOREM 8.3. Let $\{x(t), 0 \leqq t<\infty\}$ be an Ito process with state interval I, on which $\sigma>0$. Then the assertions of Theorem 8.2 remain valid under the hypothesis that $g$ is bounded in $I$.

If $I$ is compact, $g$ is of course necessarily bounded, because it is continuous. We adopt the convention that, if $r$ is an end point of the state interval but is not in the interval, then if, for some $t$, the random variable $x(t)$ has the value $r$ with positive probability, the value $g(r)$, which is involved in the assertions of the theorem, is defined as $\lim _{s \rightarrow r} g(s)$. It will be proved that this limit exists, in this case. With this convention, the processes in (8.3) are well defined.

Let $\left\{I_{n}, n \geqq 1\right\}$ be a monotone sequence of compact intervals, whose union is the state interval $I$, and suppose that each interval contains any end point of $I$ which is contained in $I$. Let $\left\{x_{n}(t), 0 \leqq t<\infty\right\}$ be the $x(t)$ process stopped on the boundary of $I_{n}$. We take this to imply that $x_{n}(t, \omega)=x(0, \omega)$ for all $t$ if $x(0, \omega) \notin I_{n}$. The theorem is proved by applying Theorem 8.2 to the $x_{n}(t)$ process and then letting $n \rightarrow \infty$, but there are various details which require some care.

Proof in case (a). If $D g=0$ in $I$, and if $g$ is bounded, $g$ can be defined by continuity at any end point of $I$ not in $I$, since $g$ is monotone. (The differential 
equation can be solved explicitly to verify the monotoneity.) In accordance with our convention, we shall therefore assume that $g$ is defined at both end points of $I$, and is continuous. According to Theorem 8.2, the $g\left[x_{n}(t)\right]$ process is a martingale. Hence, applying Theorem 1.3, with $z_{n}(t)=g\left[x_{n}(t)\right]$, the $g[x(t)]$ process is a martingale. If $D f=\Psi \geqq 0$ in $I$, and if $s$ is an interior point of $I$,

$$
f^{\prime}(\xi)=\phi(\xi) f^{\prime}(s)+\phi(\xi) \int_{0}^{\xi} \frac{2 \Psi(\eta)}{\sigma(\eta)^{2} \phi(\eta)} d \eta
$$

where

$$
\phi(\xi)=\exp \left[-2 \int_{s}^{\xi} \frac{m(\eta)}{\sigma(\eta)^{2}} d \eta\right] .
$$

Hence $f^{\prime}(\xi) \geqq 0$ for $\xi \geqq s$ if $f^{\prime}(s)>0$. Thus either $f^{\prime}$ is nondecreasing throughout $I$ or there is one point in $I$ to the left of which $f$ is monotone nonincreasing and to the right of which $f$ is monotone nondecreasing. Then if $g$ is bounded it has a limit at an end point of $I$ not in $I$, and the above argument goes through with unessential changes to prove that the $g[x(t)]$ process is a semimartingale if $D g \geqq 0$ and if $g$ is bounded in $I$.

Proof in case (b). If $D g=\lambda g$, and if $\lambda>0, g$ can have neither a positive maximum nor a negative minimum. Hence $g$ must be monotone near a boundary point of $I$, and, if bounded, can be defined by continuity at any end point of $I$ not in $I$. The method of proof of the theorem in case (a) now carries over into this case. If $\lambda=0$, this case is the same as case (a). If $\lambda<0$, $D g=\lambda g$, and if $g$ is bounded, it is not necessarily true that $g$ can be defined by continuity at an end point of $I$ not in $I$. If almost no trajectory reaches such an end point, this does not matter, and the method of proof of case (a) is applicable. This method of proof will also be applicable if, when $r$ is an end point of $I$ not in $I$, such that there is positive probability that trajectories meet this end point, it follows that $g$ has a finite limit at $r$. We prove this conclusion, as follows. According to Theorem 8.2, the process

$$
\left\{e^{-\lambda \tau_{t}(n)} g\left[x_{n}(t)\right], 0 \leqq t<\infty\right\}
$$

is a martingale, where $\tau_{t}(n)$ is the time a trajectory first meets the boundary of $I_{n}$, or $t$, whichever is the smaller. Choose $b$ so large that there is positive probability for an $x(t)$ process trajectory to reach the end point $r$ by time $b$. If we consider the above martingales for $t<b$, we can apply Theorem 1.2, and find that $\lim _{t \uparrow}, g[x(t)]$ exists almost everywhere on the set $\{\tau(\omega)<b\}$. But this implies that $\lim _{s \rightarrow r} g(s)$ exists, as was to be proved. This method is also applicable when $D g \geqq \lambda g$ to prove the result stated in the theorem, for $\lambda$ of either sign.

Proof in case (c). The method of case (a) is applicable here. If $\lambda>0[\lambda<0]$, 
$g$ can have no maximum [minimum], and it follows that if $g$ is bounded, it can be defined by continuity at any point of $I$ not in $I$.

The proof of Theorem 8.3 is now complete. The hypothesis of boundedness of $g$ in this theorem is somewhat unfortunate, but will not be a hindrance in the applications. The general idea of the proof of this theorem can be used to derive others of the same type without the boundedness hypothesis, however. Theorem 8.1 is an example of such a theorem, obtained in another way.

9. The sample functions of Ito processes. If $\{x(t), 0 \leqq t<\infty\}$ is an Ito process, we shall sometimes write $x_{\xi}(t)$ for $x(t)$ if $x(0)=\xi$, and we shall denote $\lim _{t \rightarrow \infty} x(t, \omega)$ by $x(\infty, \omega)$ whenever this limit exists. If $x\left(t_{0}, \omega_{0}\right)$ is a boundary point of the state interval, then $x\left(t, \omega_{0}\right)=x\left(t_{0}, \omega_{0}\right)$ for $t \geqq t_{0}$, and we shall say that the trajectory corresponding to $\omega_{0}$ reaches the boundary of the state interval, omitting the words "in finite time" that are sometimes used to add flavor but not meaning to the assertion. We shall see that $x\left(\infty, \omega_{0}\right)$ may be a boundary point of the state interval even though the trajectory corresponding to $\omega_{0}$ does not reach the boundary.

In the theorems of this section, we shall always be concerned with an Ito process with specified state interval. To avoid repetition we shall not repeat the full hypothesis in each theorem, but merely specify the state interval. We recall that a state interval may be infinite, but by definition it contains only finite points. On the other hand, the sample functions of an Ito process may be infinite-valued with positive probability, even at a specified value of the parameter.

Theorem 9.1 (Compact State Interval $I$ ). If $\sigma$ is positive on $I$, almost every trajectory reaches the boundary of $I$.

Let $g$ be a nonconstant function on $I$, with $D g=0$ there. Then by Theorem 8.2 , the $g[x(t)]$ process is a martingale. Since $g$ is bounded, and since the sample functions of this process are almost all continuous, it follows that these sample functions converge, when $t \rightarrow \infty$, according to a standard martingale theorem, that is, $\lim _{t \rightarrow \infty} g[x(t)]$ exists with probability 1 . In fact, according to this theorem, if this limit is assigned to the value $t=\infty$, the $g[x(t)]$ process becomes a martingale on the interval $[0, \infty]$. Since $g$ is strictly monotone, $\lim _{t \rightarrow \infty} x(t)$ exists with probability 1 . Lemma 2.4 implies that $x(\infty, \omega)$ must be an end point of $I$, for almost all $\omega$. Thus almost all trajectories converge to one or the other end point of $I$. Let $I_{1}$ be a compact interval with $I$ in its interior. Then it is no restriction on the diffusion coefficients to assume that the domain of definition of these two functions is $I_{1}$, that they satisfy the conditions IPs on this larger interval, and that $\sigma$ is positive there. Then we can assume that the $x(t)$ process is obtained by stopping an $x_{1}(t)$ process with state interval $I_{1}$. Since the latter process trajectories almost all have as limits the end points of $I_{1}$, they must pass through one or the other end point of $I$, that is, almost every $x(t)$ process trajectory reaches the boundary of $I$. 
Theorem 9.2 (State Interval $I:\left[r_{1}, r_{2}\right)$ ). If $\sigma$ is positive on $I, \lim _{t \rightarrow \infty} x(t)$ $=x(\infty)$ exists with probability 1 , and $x(\infty, \omega)$ is almost always either $r_{1}$ or $r_{2}$ (depending on $\omega$ ). For almost all $\omega$ with $x(\infty, \omega)=r_{1}, x(t, \omega)=r_{1}$ for sufficiently large $t$.

Let $g$ be defined as in the proof of the preceding theorem. Then $g$ need no longer be bounded, but is bounded from above or below, depending on whether it is monotone decreasing or increasing. Replacing $g$ by a linear function of $g$, we can assume that $g$ is non-negative and increasing. In proving the theorem, it is no restriction to assume that $x(0)=\xi$ is a constant, and we shall do so. Let $\left\{x_{n}(t), 0 \leqq t<\infty\right\}$ be the $x(t)$ process stopped at $r_{2}-1 / n$ if $r_{2}$ is finite, or at $n$ if $r_{2}=\infty$, where we choose $n$ so large that $\xi$ lies in the diminished state interval. Applying the preceding theorem to the $x_{n}(t)$ process, we find that, excluding a set of trajectories of probability 0 , an $x(t)$ process trajectory either goes to $r_{1}$ and stops, goes to $r_{2}$ and stops, approaches $r_{2}$ as a limit without reaching that point, or does not approach a limit. The latter case will now be ruled out (with probability 1) by an application of Theorem 1.2 to the processes $\left\{g\left[x_{n}(t)\right], 0 \leqq t<\infty\right\}$. These processes are martingales, according to Theorem 8.2. The random variables of these martingales are non-negative, and hence

$$
E\left\{\left|g\left[x_{n}(t)\right]\right|\right\}=E\left\{g\left[x_{n}(0)\right]\right\}=g(\xi)
$$

is independent of both $t$ and $n$. Hence, according to Theorem 1.2, whose other hypotheses are obviously satisfied, $\lim _{t \rightarrow \infty} g[x(t)]$ exists and is finite with probability 1 . Since $g$ is monotone, it follows that $\lim _{t \rightarrow \infty} x(t)$ exists with probability 1 , and the proof of the theorem is now complete.

Theorem 9.3 (Compact STATE INTERVAL $I:\left[r_{1}, r_{2}\right]$ ). If $\sigma>0$ on $I$, and if $D g=0$ there, then

$$
E\{g[x(\infty)]\}=E\{g[x(0)]\} .
$$

If the function $p_{i}$ is defined on $I$ by

$$
p_{i}(\xi)=P\left\{x_{\xi}(\infty, \omega)=r_{i}\right\},
$$

then $p_{i}$ is the unique solution on $I$ of

$$
D f=0, \quad f\left(r_{i}\right)=1, \quad f\left(r_{i}\right)=0
$$

We remarked in the course of the proof of Theorem 9.1 that the $g[x(t)]$ process is a martingale on the parameter interval $[0, \infty]$. The equality (9.1) simply expresses the equality of expectations at $t=0, \infty$ for this martingale. (See the remarks on the classical ruin problem at the beginning of §8.) If $x(0)=\xi$, the two sides of (9.1) can be evaluated explicitly, and we obtain

$$
p_{1}(\xi) g\left(r_{1}\right)+p_{2}(\xi) g\left(r_{2}\right)=g(\xi) .
$$


In particular, if $g$ is the special solution described in the theorem, this equation states that $g(\xi)=p_{i}(\xi)$. Note that the fact that the solution of the differential equation with the specified boundary condition is unique is proved by the above identification, making unnecessary any uniqueness theorem obtained from a study of the differential equation (trivial in this case, of course). Note also how the problem of proving the existence of derivatives of $p_{i}$ directly was carefully avoided.

Let $m, \sigma$ be continuous functions defined in the interval $\left[r_{1}, r\right)$, where $r_{1}<r \leqq \infty$, with $\sigma>0$. Then we shall say that the condition $A(r)$ is satisfied to the left of $r$ if the function $\phi$ defined by

$$
\phi(\xi)=\exp \left[-2 \int_{r_{1}}^{\xi} \frac{m(\eta)}{\sigma(\eta)^{2}} d \eta\right]
$$

is integrable over $\left[r_{1}, r\right)$. We shall say that condition $B(r)$ is satisfied to the left of $r$ if $A(r)$ is satisfied and if

$$
\phi(\xi) \int_{r_{1}}^{\xi} \frac{d \eta}{\sigma(\eta)^{2} \phi(\eta)}
$$

is integrable over $\left[r_{1}, r\right)$. The corresponding definitions to the right of $r$, with $-\infty \leqq r<\infty$, are obtained by making $r_{1}>r$ above. The specification of the side will be omitted whenever it is obvious.

In the terminology of Feller [5], thinking of $m, \sigma$ as diffusion coefficients of an Ito process with $r$ as end point of the state interval, the condition $B(r)$ is the condition that $r$ be a regular or exit boundary. We shall say that condition $C(r)$ is satisfied on the left if $A(r)$ is not satisfied, and if

$$
\frac{1}{\sigma(\xi)^{2} \phi(\xi)} \int_{r_{1}}^{\xi} \phi(\eta) d \eta
$$

is integrable on $\left[r_{1}, r\right)$ (Feller's entrance boundary). If neither $B(r)$ nor $C(r)$ is satisfied, we shall say that condition $D(r)$ is satisfied (Feller's natural boundary).

Suppose that $m$ and $\sigma$ satisfy the conditions IPs imposed on diffusion coefficients, in some state interval, and let $r$ be an end point of the state interval which is not included in it. Then, solving $D f=0$ explicitly, it is clear that the condition $A(r)$ is equivalent to the condition that every solution of the differential equation be bounded near $r$. Hille ([8], see also [5]) showed that the condition $B(r)$ is equivalent to the condition that, if $\lambda$ is a positive constant, all solutions of $D f=\lambda f$ be bounded near $r$. We have already remarked that, if a solution is bounded near $r$, the solution has a finite limit at $r$. If $\lambda<0$, it is easy to see that the condition $A(r)$ implies that every solution of $D f=\lambda f$ is bounded. In fact, if $D f=\lambda f$, and if $\lambda<0, f$ can have no positive minimum or negative maximum, and unless the function vanishes identically, 
cannot have a zero derivative when it vanishes. If a solution starts say from 0 , with a positive derivative, the solution must either increase thereafter throughout the domain of definition or increase to a maximum and then decrease, either decreasing thereafter or decreasing to a negative minimum and then increasing, and so on. Moreover the equation

$$
f^{\prime}(\xi)=\phi(\xi) f^{\prime}(s)+\lambda \phi(\xi) \int_{s}^{\xi} \frac{2 f(\eta)}{\sigma(\eta)^{2} \phi(\eta)} d \eta,
$$

where $\phi$ is defined above, shows that no increase or decrease from a zero at a point $s$ can exceed the amount

$$
\left|f^{\prime}(s)\right| \int_{s}^{r_{2}} \phi(\xi) d \xi .
$$

Hence, if condition $A(r)$ is satisfied, $f$ must be bounded near $r$. Finally, the equation $D f=\lambda$ can be solved explicitly, and the solution exhibits the fact that, if condition $B(r)$ is satisfied, the solution has a finite limit at $r$.

Theorem 9.4 (State INTERVAL $I:\left[r_{1}, r_{2}\right)$ ). If $\sigma>0$ on $I$ and if $p_{i}$ is defined by (9.2), $p_{2}(\xi)$ is positive for some $\xi$ in the interior of $I$ if and only if condition $A\left(r_{2}\right)$ is satisfied. In the latter event, if $D g=0$ on $I, p_{i}$ can be evaluated as in Theorem 9.3.

This theorem implies that $p_{2}$ is either positive everywhere in the interior of $I$ or vanishes identically there. Note that $p_{2}(\xi)$ is not the probability that a trajectory from $\xi$ reaches $r_{2}$. In fact, as we shall see in Theorem 9.6, unless condition $B\left(r_{2}\right)$ is satisfied, almost no trajectory reaches $r_{2}$, whether it approaches that point or not. As usual, we assume that $g$ in the statement of the theorem is defined by continuity at $r_{2}$ if condition $A\left(r_{2}\right)$ is satisfied.

Suppose that $D g=0$ on $I$. Then, as was proved in the course of proving Theorem 9.2, $\lim _{t \rightarrow \infty} g\left[x_{\xi}(t, \omega)\right]$ exists and is finite with probability 1. If $p_{2}(\xi)>0$, this limit must be $\lim _{r \rightarrow r_{2}} g(r)$, with probability $p_{2}(\xi)$. Thus the latter limit must exist, and it follows that $g$ is a bounded (monotone) function in $I$, so that condition $A\left(r_{2}\right)$ is satisfied. Conversely, if condition $A\left(r_{2}\right)$ is satisfied, and if $D g=0, g$ is bounded, and therefore the stochastic process $\{g[x(t)], 0 \leqq t<\infty\}$ is a martingale, by Theorem 8.3. Since $g$ is bounded, it follows from a standard martingale theorem that the process is even a martingale on the parameter interval $[0, \infty]$. This fact was all that was needed in the evaluation of $p_{i}$ given in Theorem 9.3. In this evaluation, $p_{i}$ is strictly monotone, and therefore never vanishes in the interior of the state interval. Note that in this evaluation $D f$ is to vanish only on $I$, which does not now include the point $r_{2}$. The point $r_{2}$ may be infinite, and, even if $r_{2}$ is finite, $f$ may not have two derivatives there.

It is not yet clear how to obtain the distribution of the time needed to reach the boundary of the state interval, or whether trajectories converging 
to an end point of the state interval not in the interval will necessarily reach it. The following two theorems answer these questions.

Theorem 9.5 (Compact STATE INTERVAL $I:\left[r_{1}, r_{2}\right]$ ). Suppose that $\sigma>0$ in I. Let $\tau\left[{ }_{i} \tau\right]$ be the time a trajectory first meets the boundary of $I$ [the point $r_{i}$ ], or $+\infty$ if there is no such meeting, and let $\lambda$ be any positive constant. Then, if $D g=\lambda g$ on $I$,

$$
E\left\{e^{-\lambda \tau} g[x(\infty)]\right\}=g\left(r_{1}\right) E\left\{e^{-\lambda_{1} \tau}\right\}+g\left(r_{2}\right) E\left\{e^{-\lambda_{2} \tau}\right\}=E\{g[x(0)]\} .
$$

If $x(0)=\xi, E\left\{e^{-\lambda_{i} r}\right\}$ is the unique solution on $I$ of

$$
D f=\lambda f, \quad f\left(r_{j}\right)=0, \quad f\left(r_{i}\right)=1 \quad(j \neq i) .
$$

According to Theorem 8.2 , if $D g=\lambda g$, the stochastic process (8.5b) is a martingale. In the present case, $\lambda>0$, so that the random variables of this martingale are uniformly bounded, and have the probability 1 limit $g[x(\infty)]$ when $t \rightarrow \infty$. It follows from a standard martingale theorem that the martingale is even a martingale on the parameter set $[0, \infty]$. The equation (9.3) simply expresses the fact that the expectations of the random variables of this martingale are equal for the two parameter values $0, \infty$. A solution of $D f=\lambda f$ has no positive maximum or negative minimum. It then follows that there is one and only one solution $g$ with $g\left(r_{i}\right)=0, g\left(r_{j}\right)=1$. If $x(0)=\xi$, and if this solution is used in (9.3), we find that

$$
E\left\{e^{-\lambda_{i} \tau}\right\}=g(\xi) .
$$

The theorem is now completely proved. The characterization of the solution of $g$ given in (9.4) could have been used to prove that $g$ was unique. Note that (9.4) can be put in the form

$$
\int_{0}^{\infty} e^{-\lambda t} d_{t} P\left\{{ }_{i} \tau(\omega) \leqq t\right\}=\lambda \int_{0}^{\infty} e^{-\lambda t} P\left\{{ }_{i} \tau(\omega) \leqq t\right\}=g(\xi) .
$$

Thus the equation provides an explicit expression of the Laplace transform of the distribution function of ${ }_{i} \tau$.

The restriction that $\lambda$ be positive in Theorem 9.5 can be weakened. We first remark that there is a positive solution of $D f=\lambda f$ in $I$, if $|\lambda|$ is sufficiently small, because there is a positive solution of $D f=0$. If $g$ is such a solution of the former equation, $g(\xi)=E\left\{e^{-\lambda \tau} g\{[x(t)]\}\right.$, since the process (8.5b) is a martingale. When $t \rightarrow \infty$, we find that $E\left\{e^{-\lambda r}\right\}$ is finite. Thus there is an open interval $(-\delta, 0), \delta>0$, of values of $\lambda$ for which this expectation is finite. If $\lambda$ is in this interval, the proof of Theorem 9.5, with minor modifications, remains valid.

Theorem 9.6 (State interval $I:\left[r_{1}, r_{2}\right)$ ). Suppose that $\sigma>0$ in I. If condition $B\left(r_{2}\right)$ is satisfied, almost every trajectory of the process with $x(\infty, \omega)$ $=r_{2}$ meets $r_{2}$, and the evaluations of Theorem 9.5 are valid. Conversely, if, for 
some $\xi \in\left(r_{1}, r_{2}\right)$, there is positive probability that a trajectory from $\xi$ meets $r_{2}$, then condition $B\left(r_{2}\right)$ is satisfied.

If $D g=\lambda g(\lambda>0)$ in $I$, and if condition $B\left(r_{2}\right)$ is satisfied, then $g$ is bounded, and the stochastic process $\left\{e^{-\lambda \tau_{t} g}[x(t)], 0 \leqq t \leqq \infty\right\}$ is a martingale, by exactly the same reasoning as in the preceding theorem's proof. Then the evaluations of the preceding theorem, which are simply translations of the martingale property for this process, are valid. In particular, if $g^{\prime}\left(r_{1}\right)>0, g$ is monotone increasing in $I$, since $g$ has no positive maximum. But then (9.3) with this $g$, and with $x(0)=\xi$, implies that ${ }_{1} \tau$ and ${ }_{2} \tau$ must be finite with positive probability. Since Theorem 9.4 is applicable, $p_{2}(\xi) \rightarrow 1$ when $\xi \rightarrow r_{2}$. Hence, when the initial position $\xi$ goes to $r_{2}$, the first term on the left in (9.3) (in the middle of the three expressions in this continued equality) drops out, and the probability that ${ }_{2} \tau$ is finite must approach 1 . If $x(0)=\xi$ is fixed, a trajectory which approaches $r_{2}$ as a limit when $t \rightarrow \infty$ must reach every point $\xi_{1}$ between $\xi$ and $r_{2}$, and, if $\xi_{1}$ is near $r_{2}$, must have probability near 1 of reaching $r_{2}$ in finite time once it has reached $\xi_{1}$. Thus almost every trajectory from $\xi$ which approaches $r_{2}$ as a limit must reach it, as was to be proved. Conversely, suppose that there is positive probability of reaching $r_{2}$ from some $\xi$. Then condition $A\left(r_{2}\right)$ is satisfied, according to Theorem 9.4. Now suppose that $D g=\lambda g$ on $I$ for some positive $\lambda$, and let $\left\{x_{n}(t), 0 \leqq t<\infty\right\}$ be the Ito process stopped at $r_{2 n}$. Here $\left\{r_{2 n}, n \geqq 1\right\}$ is any sequence in $\left(r_{1}, r_{2}\right)$ converging monotonely to $r_{2}$. The time ${ }_{j} \tau$ becomes ${ }_{j} \tau_{n}$ for the stopped process. Applying Theorem 9.5 to the $x_{n}(t)$ process with $x_{n}(0)=\xi$, as is possible if $n$ is sufficiently large, we find that

$$
g(\xi)=g\left(r_{1}\right) E\left\{e^{-\lambda_{1} r_{n}}\right\}+g\left(r_{2 n}\right) E\left\{e^{-\lambda 2^{r_{n}}}\right\} .
$$

When $n \rightarrow \infty$, the first term on the right becomes $g\left(r_{1}\right) E\left\{e^{-\lambda_{1} \tau_{n}}\right\}$. The second expectation on the right has the (nonzero) limit $E\left\{e^{-\lambda_{2} \tau_{n}}\right\}$. Hence $\lim _{n \rightarrow \infty} g\left(r_{2 n}\right)$ exists and is finite, and this implies that $g$ is bounded. Thus every solution of $D f=\lambda f(\lambda>0)$ is bounded, that is, condition $B\left(r_{2}\right)$ is satisfied.

Even if $B\left(r_{2}\right)$ is not satisfied, there is still $[5 ; 8]$ a bounded non-negative monotone nonincreasing solution of $D f=\lambda f(\lambda>0)$. In this case the time $\tau$ to the boundary becomes the time $(\leqq \infty)$ to the boundary point $r_{1}$. Theorem 9.5 is extended as follows.

Theorem 9.7 (State interval $I:\left[r_{1}, r_{2}\right)$ ). Suppose that $\sigma>0$ on $I$ and that condition $B\left(r_{2}\right)$ is not satisfied. Then, if $x(0)=\xi, E\left\{e^{-\lambda_{1} r}\right\}(\lambda>0)$ is the unique bounded solution on $I$ of

$$
D f=\lambda f, \quad f\left(r_{1}\right)=1 .
$$

In fact, if $g$ is bounded on $I$, and if $D g=\lambda g$, the process $\left\{e^{-\lambda \tau} g[x(t)]\right.$, $0 \leqq t \leqq \infty\}$ is a martingale, just as in the proof of the preceding theorem. Setting expectations equal at the parameter values $0, \infty$ we find that 


$$
g(\xi)=E\left\{e^{-\lambda \tau} g[x(\infty)]\right\}=g\left(r_{1}\right) E\left\{e^{-\lambda \tau}\right\},
$$

and this yields the stated result. The fact that (9.6) together with the condition that $f$ be bounded determines $f$ uniquely follows either from the theory of the operator $D$ or from the evaluation we have given of the probability significance of the solution.

Theorem 9.7 enables us to give the following rough description of the increase of $\tau_{1}$ as the initial position $\xi$ approaches $r_{2}$, as follows.

CoROLlaRy. In Theorem 9.7, if condition $C\left(r_{2}\right)$ is satisfied, there is a distribution function $F$, such that

$$
\lim _{\xi \rightarrow r_{2}} P\left\{{ }_{1} \tau(\omega) \leqq \alpha\right\}=F(\alpha)
$$

at all continuity points of $F$. If condition $D\left(r_{2}\right)$ is satisfied,

$$
\lim _{\xi \rightarrow r_{2}} P\left\{{ }_{1} \tau(\omega) \leqq \alpha\right\}=0
$$

for every $\alpha$.

If condition $C\left(r_{2}\right)$ is satisfied, let $g$ be a solution of (9.6). Then it is known [5] that $g$ has a positive limit at $r_{2}$. In other words

$$
\lim _{\xi \rightarrow r_{2}} E\left\{e^{-\lambda_{1} r}\right\}
$$

exists for every $\lambda>0$, and is a positive number less than 1 . To prove the first statement of the corollary we need only prove that, roughly, as $\xi$ varies, the distribution of ${ }_{1} \tau$ is uniformly finite, more precisely that

$$
\lim _{\alpha \rightarrow \infty} \liminf _{\xi \rightarrow r_{2}} P\left\{{ }_{1} \tau(\omega) \leqq \alpha\right\}=1 .
$$

To prove (9.9), choose $r_{1}^{\prime}, \xi$, with $r_{1}<r_{1}^{\prime}<\xi<r_{2}$. The time a trajectory takes from $\xi$ to $r_{1}$ is the sum of the time from $\xi$ to $r_{1}^{\prime}$ and the time from $r_{1}^{\prime}$ to $r_{1}$. Since the latter time is finite and independent of the former, the left side of (9.9) is unchanged if $r_{1}$ is replaced in the whole discussion by $r_{1}^{\prime}$. But this change replaces $g$ by $g / g\left(r_{1}^{\prime}\right)$. Thus, for fixed $\lambda$, we can choose $r_{1}^{\prime}$ so close to $r_{2}$ that the limit in (9.8), which is now $\lim _{r \rightarrow r_{2}} g(r) / g\left(r_{1}^{\prime}\right)$, is as close to 1 as desired. This fact obviously implies (9.9). To prove (9.7) under condition $D\left(r_{2}\right)$, we need only remark that the condition is known to make 0 the limiting value at $r_{2}$ of a bounded solution of (9.6) [5]. Hence the limit in (9.8) is 0 , and this implies (9.7).

We now apply case (c) of Theorem 8.2. Since $D(\lambda f)=\lambda$ when $D f=1$, we can and shall choose $\lambda=-1$ without loss of generality. We use the notation of Theorems 9.3 and 9.5 .

Theorem 9.8 (Compact State Interval $I:\left[r_{1}, r_{2}\right]$ ). Suppose that $\sigma>0$ 
on I. If $D g=-1$ on $I$,

$$
E\{\tau\}=E\{g[x(0)]\}-E\{g[x(\infty)]\} .
$$

If $x(0)=\xi, E\{\tau\}$ is the unique solution on $I$ of

$$
D f=-1, \quad f\left(\boldsymbol{r}_{1}\right)=f\left(\boldsymbol{r}_{2}\right)=0 .
$$

According to Theorem 8.2, if $D g=-1$ on $I$, the stochastic process (8.5c) with $\lambda=-1$ is a martingale. Hence

$$
E\{g[x(t)]\}-E\left\{\tau_{t}\right\}=E\{g[x(0)]\} .
$$

Since $\tau_{t}$ is nondecreasing when $t$ increases, since $\lim _{t \rightarrow \infty} g[x(t)]=g[x(\infty)]$ with probability 1 , and since $g$ is bounded, it follows that $E\{\tau\}$ exists, and that (9.10) is true. The random variables of the martingale $(8.5 \mathrm{c})$ are dominated by an integrable function, the sum of $\tau$ and a bound of $g$. Hence the martingale is even a martingale on the enlarged parameter interval $[0, \infty]$. The equality (9.10) is simply a statement of the equality of expectations in this martingale for $t=0, \infty$. If $x(0)=\xi,(9.10)$ becomes

$$
E\{\tau\}=g(\xi)-p_{1}(\xi) g\left(r_{1}\right)-p_{2}(\xi) g\left(r_{2}\right)
$$

and, in view of the characterization of $p_{i}$ obtained in Theorem 9.3, $E\{\tau\}$ is indeed a solution of the stated differential equation with the specified boundary condition. To prove uniqueness without recourse to differential equation theory we need only remark that, if $g$ is a solution of $(9.11),\left(9.10^{\prime}\right)$ becomes $E\{\tau\}=g(\xi)$.

Theorem 9.9 (State interval $I:\left[r_{1}, r_{2}\right)$ ). Suppose that $\sigma>0$ on I. If condition $B\left(r_{2}\right)$ is satisfied, the evaluations of Theorem 9.8 are valid. If condition $B\left(r_{2}\right)$ is not satisfied, suppose that $x(0)=\xi$. Then $E\{\tau\}$ is finite if and only if $A\left(r_{2}\right)$ is not satisfied and

$$
\int_{r_{1}}^{r_{2}} \frac{2 d \eta}{\sigma(\eta)^{2} \phi(\eta)}=c<\infty \quad \text { where } \phi(\xi)=\exp \left[-2 \int_{r_{1}}^{\xi} \frac{m(\eta)}{\sigma(\eta)^{2}} d \eta\right] .
$$

Under these hypotheses, $E\{\tau\}$ is the unique solution of

$$
D f=-1, \quad f\left(r_{1}\right)=0, \quad f^{\prime}\left(r_{1}\right)=c .
$$

This solution is monotone increasing. It is bounded if and only if (9.12) is strengthened to

$$
\int_{r_{1}}^{r_{2}} \frac{2 \Phi(\eta) d \eta}{\sigma(\eta)^{2} \phi(\eta)}<\infty \quad \text { where } \Phi(\xi)=\int_{r_{1}}^{\xi} \phi(\eta) d \eta
$$

(that is, if and only if $C\left(r_{2}\right)$ is satisfied).

If condition $B\left(r_{2}\right)$ is satisfied, the equation $D f=-1$ has only bounded solutions, as can be seen from the explicit solutions. Then in this case, if 
$D g=-g$, the stochastic process $\left\{g[x(t)]+\tau_{t}, 0 \leqq t<\infty\right\}$ is a martingale, by Theorem 8.3, where $g$ is defined at $r_{2}$ by continuity. Since $g$ is bounded, it follows that this stochastic process remains a martingale if $\infty$ is added to the parameter set, and this fact yields the evaluations of Theorem 9.8, just as in the case of a compact state interval.

If condition $B\left(r_{2}\right)$ is not satisfied, $\tau={ }_{1} \tau$ with probability 1 . If $A\left(r_{2}\right)$ is not satisfied either, then $\tau={ }_{1} \tau<\infty$, with probability 1 , and this is the only case when $B\left(r_{2}\right)$ is not satisfied and $E\{\tau\}$ can be finite. In the following, we shall suppose throughout that $A\left(r_{2}\right)$ is not satisfied and that $x(0)=\xi$. Let $\left\{r_{2 n}, n \geqq 1\right\}$ be a monotone sequence of points in the state interval, with limit $r_{2}$, let $\left\{x_{n}(t), 0 \leqq t<\infty\right\}$ be the $x(t)$ process stopped at $r_{2 n}$, and let $\tau(n)$ be the corresponding stopping time. If $D g=-1$, and if $n$ is so large that $r_{2 n}>\xi$, we have, from the preceding theorem, $E\{\tau(n)\}=g(\xi)-E\left\{g\left[x_{n}(\infty)\right]\right\}$. Since $\tau(n) \uparrow \tau$ with probability 1 when $n \rightarrow \infty$,

$$
E\{\tau\}=g(\xi)-\lim _{n \rightarrow \infty} E\left\{g\left[x_{n}(\infty)\right]\right\} .
$$

Now if $D f=-1$,

$$
f^{\prime}(\xi)=\phi(\xi) f^{\prime}\left(r_{1}\right)-\phi(\xi) \int_{r_{1}}^{\xi} \frac{2 d \eta}{\sigma(\eta)^{2} \phi(\eta)},
$$

where $\phi$ is defined in (9.12). Hence, since condition $A\left(r_{2}\right)$ is not satisfied, that is, since $\phi$ is not integrable on $\left[r_{1}, r_{2}\right), f$ cannot be bounded unless $f^{\prime}\left(r_{1}\right)>0$, (9.12) is satisfied, and $f^{\prime}\left(r_{1}\right)=c$. Conversely, if these conditions are satisfied, (9.16) becomes

$$
f^{\prime}(\xi)=\phi(\xi) \int_{\xi}^{r 2} \frac{2 d \eta}{\sigma(\eta)^{2} \phi(\eta)}
$$

and $f$ is then bounded if and only if the right side of $\left(9.16^{\prime}\right)$ is integrable. This integrability condition is equivalent to (9.14). There is always an unbounded solution of $D f=-f$, for example any solution with $f^{\prime}\left(r_{1}\right) \neq c$ when (9.12) is satisfied, any solution whatever when (9.12) is not satisfied. If we let $g$ in (9.15) be an unbounded solution, we find, using Theorem 9.3 and (9.16),

$$
\begin{aligned}
E\{\tau\} & =g(\xi)-\lim _{n \rightarrow \infty}\left[g\left(r_{1}\right) P\left\{x_{n}(\infty, \omega)=r_{1}\right\}+g\left(r_{2 n}\right) P\left\{x_{n}(\infty, \omega)=r_{2 n}\right\}\right] \\
& =g(\xi)-g\left(r_{1}\right)-\Phi(\xi) \lim _{n \rightarrow \infty} \frac{g\left(r_{2 n}\right)}{\Phi\left(r_{2 n}\right)} \\
& =g(\xi)-g\left(r_{1}\right)-\Phi(\xi) \lim _{n \rightarrow \infty} \frac{g^{\prime}\left(r_{2 n}\right)}{\phi\left(r_{2 n}\right)} \\
& =g(\xi)-g\left(r_{1}\right)-\Phi(\xi)\left[g^{\prime}\left(r_{1}\right)-\int_{r_{1}}^{r_{2}} \frac{2 d \eta}{\sigma(\eta)^{2} \phi(\eta)}\right] .
\end{aligned}
$$


Thus $E\{\tau\}$ is finite if and only if (9.12) is satisfied, and in that case $E\{\tau\}$ satisfies (9.13). Conversely, if (9.12) is satisfied, and if $g$ satisfies (9.13) and is unbounded, (9.17) is applicable, and yields

$$
E\{\tau\}=g(\xi),
$$

whereas, if $g$ is bounded, (9.15) yields this same evaluation immediately. The first assertion of the theorem has now been proved. We have already seen that if $g$ satisfies (9.13) it will be bounded if and only if (9.14) is true, and this fact implies the truth of the last assertion of the theorem.

The preceding theorems make it easy to investigate the character of the sample functions of Ito processes with open state intervals, and the results will now be sketched without proofs. Let the state interval be $\left(r_{1}, r_{2}\right)$, where the end points may be finite or infinite, and suppose that $\sigma>0$ on the state interval.

If neither $A\left(r_{1}\right)$ nor $A\left(r_{2}\right)$ are satisfied, almost all trajectories are oscillatory, as $t \rightarrow \infty$, with both $r_{1}$ and $r_{2}$ as limit points. The Brownian motion process, in which $r_{1}=-\infty, r_{2}=\infty$, is an example of this case.

If $A\left(r_{1}\right)$ is satisfied, but if neither $B\left(r_{1}\right)$ nor $A\left(r_{2}\right)$ is satisfied, almost all trajectories approach $r_{1}$, without ever reaching it.

If $B\left(r_{1}\right)$ is satisfied, but if $A\left(r_{2}\right)$ is not, almost all trajectories reach $r_{1}$.

If $A\left(r_{1}\right)$ and $A\left(r_{2}\right)$ are satisfied, but neither $B\left(r_{1}\right)$ nor $B\left(r_{2}\right)$, almost all trajectories from any initial point $\xi$ approach either $r_{1}$ or $r_{2}$ asymptotically, without ever reaching it, and the probabilities of the two cases can be calculated exactly as in Theorem 9.2. If $A\left(r_{i}\right)$ is strengthened to $B\left(r_{i}\right)$, almost all trajectories approaching the point $r_{i}$ reach it.

If $B\left(r_{1}\right)$ is satisfied, but if $B\left(r_{2}\right)$ is not, then, using the usual notation, if $x(0)=\xi, E\left\{e^{-\lambda \tau}\right\}$ and $E\{\tau\}$ are characterized exactly as in Theorems 9.7 and 9.9. If $B\left(r_{1}\right)$ and $B\left(r_{2}\right)$ are both satisfied, Theorems 9.5 and 9.8 describe the appropriate evaluations of these expectations.

We have omitted the usual converses in this outline, but they hold, just as in earlier work. For example, if, for some $\xi$, there is positive probability that a trajectory from $\xi$ meets $r_{1}$, then $B\left(r_{1}\right)$ is satisfied.

We shall now study the character of Ito process sample functions in the neighborhood of isolated zeros of the diffusion coefficient $\sigma$. We first make the following rather trivial remark. If $\xi$ is an interior point of the state interval, and if $m(\xi)=\sigma(\xi)=0$, then almost no trajectory with initial point $\xi$ ever leaves that point. To prove this we can extend the definition of the diffusion coefficients from a neighborhood of $\xi$ to the whole line and assume that the process is a standard Ito process. If now the equation (2.1) with $x(0)=\xi$ is solved by successive approximation, with first approximation $\xi$, we find that all approximations are identically $\xi$, so that the trajectories from $\xi$ stay at $\xi$, as was to be proved. It follows that a trajectory from one side of $\xi$ almost never passes to the other. Either it does not reach $\xi$ or it reaches $\xi$ and stays there. The fol- 
lowing theorem extends this result by dropping the hypothesis that $m$ vanish at $\xi$. It is obviously sufficient to consider the case $m(\xi)<0$.

Theorem 9.10. Let $\{x(t), 0 \leqq t<\infty\}$ be an Ito process, and let $\xi$ be an interior point of its state interval. Suppose that $\xi$ is an isolated zero of $\sigma$, and that $m(\xi)<0$. Then almost every trajectory with initial point $\xi$ leaves $\xi$ at once, entering the interval $(-\infty, \xi)$, and neither returns to $\xi$ nor approaches $\xi$ asymptotically.

There is no restriction in assuming that the Ito process is a standard one, that $\sigma$ never vanishes in some interval $(\xi-\delta, \xi+\delta)$, and that both diffusion coefficients vanish identically outside this interval. Since $m(\xi)<0$, condition $B(\xi)$ is satisfied on the right, and it is easily verified that condition $C(\xi)$ is satisfied on the left, using the fact that, according to our hypotheses on the diffusion coefficients, $\sigma$ must vanish to the first order at $\xi$. It follows that, if a trajectory ever reaches a point $\xi_{1}<\xi$, it never returns to $\xi$, and does not even converge asymptotically to $\xi$. Moreover, we now show that no trajectory from $\xi$ ever reaches any point $\xi_{2}>\xi$. In fact, for any $t_{0}>0$, the trajectories from $\xi$ are, with high probability, uniformly on $\left[0, t_{0}\right]$ close to those from $\xi_{1}<\xi$ if $\xi_{1}$ is near $\xi$, by Lemma 2.2. Since almost no trajectory from $\xi_{1}$ every reaches $\xi$, it follows that, if $\epsilon>0$, the probability that a trajectory from $\xi$ will ever reach $\xi+\epsilon$ in time $\leqq t_{0}$ is $<\epsilon$. Then almost no trajectory from $\xi$ ever reaches any point to the right of $\xi$. There is, however, positive probability of reaching points to the left of $\xi$, since

$$
E\left\{x_{\xi}(t)-\xi\right\}=m(\xi) t+O\left(t^{3 / 2}\right) .
$$

The probability that a trajectory from $\xi$ remains at $\xi$ for time $t$ satisfies the functional equation

$$
f\left(t_{1}+t_{2}\right)=f\left(t_{1}\right) f\left(t_{2}\right) \quad\left(t_{1}, t_{2} \geqq 0\right),
$$

with $0 \leqq f(t)<1$. Hence $f(t)=e^{-a t}$ for some $a, 0<a \leqq \infty$. Using the fact that almost no trajectory from $\xi$ ever leaves the interval $[\xi-\delta, \xi+\delta]$, an elementary calculation yields the inequality

$$
-m(\xi) t-\alpha t+O\left(t^{3 / 2}\right)<\delta P\left\{x_{\xi}(t, \omega)-\xi \leqq-\alpha t\right\} \leqq \delta\left(1-e^{-a t}\right) .
$$

It follows that, if $a$ is finite,

$$
-m(\xi)-\alpha<\delta a,
$$

where this inequality must hold for all positive $\alpha, \delta$. Since this domain of validity is impossible, $a=\infty$, and we have proved that a trajectory with initial points $\xi$ leaves that point at once. The proof of the theorem is now complete. An application of Theorem 9.9 shows that a trajectory from $\xi$ meets a point $\xi_{1}$ to the left of $\xi$ in a time $\tau$ with finite expectation if $\sigma$ does not vanish in the interval $\left[\xi_{1}, \xi\right)$.

We conclude this paper with a few remarks on the use of complex func- 
tions in the theorems of this and the preceding section. Since semimartingales are necessarily real stochastic processes, we need only consider martingale sections of the theorems, and it is to be understood that the semimartingale sections are ignored in the following remarks. The possibilities are illustrated by an examination of Theorem 8.1, on which the later theorems are based. There is no advantage in allowing $g$ to be complex in case (a), because the real and imaginary parts of $g$ would then be subsumed under case (a) for real functions. There is no advantage in allowing $\lambda$ to be complex in case (c), since $D(g / \lambda)=1$ if $D g=\lambda$. Furthermore there is no. advantage in allowing $g$ to be complex in solving $D g=\lambda$ with $\lambda$ real, because the real part of $g$ then satisfies this same equation, and the imaginary part provides a process already defined in the (real) case (a). Thus, although Theorem 8.1 is applicable to the complex field, with no change in proof, there is nothing new to be obtained except in case (b), and we shall only look for further applications of this case in studying later theorems. For example, in case (b) of Theorem 8.1, if the $x(t)$ process is a Brownian motion process, we find that the stochastic process

$$
\left\{e^{\alpha x(t)-\alpha^{2} t / 2}, 0 \leqq t<\infty\right\}
$$

is a martingale, for every complex number $\alpha$. The complex number $\lambda$ of case (b) is identified with $\alpha^{2} / 2$ here. This fact is easily deduced directly.

Theorem 8.2 case (b) goes through with no change in the complex case. Theorem 8.3 case (b) also goes through with no change, since the proof given for the case $\lambda<0$ is applicable to the complex case.

Theorems 9.5, 9.6, 9.7 are valid for $\lambda$ complex and properly restricted. For example (9.3) in Theorem 9.5 is valid (with no change in the proof) for any $\lambda$ with a non-negative real part. (We make the convention that, for such a value of $\lambda, e^{-\lambda \infty}=0$.) This equation will be used in proving the following theorem on the solutions of $D f=\lambda f$ for $\lambda$ complex. This theorem generalizes those of Hille and Feller in the real case.

THEOREM 9.11. Let $\lambda$ have a non-negative real part, and let $m, \sigma^{2}$ be diff usion coefficients satisfying conditions IPs on the state interval $\left[r_{1}, r_{2}\right)$, with $\sigma>0$ there.

(i) If $D g=\lambda g$, then $g$ vanishes identically if it has more than one zero.

(ii) There is a nontrivial bounded solution of $D f=\lambda f$.

(iii) If condition $B\left(r_{2}\right)$ or $C\left(r_{2}\right)$ is satisfied, there is a nontrivial solution of $D f=\lambda f$ with a finite limit at $r_{2}$.

(iv) If $\lambda$ has a positive real part, there is a nontrivial solution of $D f=\lambda f$ with the limit 0 at $r_{2}$.

(v) If condition $B\left(r_{2}\right)$ is satisfied, every solution of $D f=\lambda f$ has a finite limit at $r_{2}$.

Let $\{x(t), 0 \leqq t<\infty\}$ be an Ito process with the given state interval and diffusion coefficients. Let $g_{1}, g_{2}$ be nontrivial solutions of $D f=\lambda f$, with $g_{1}\left(r_{1}\right)$ $=0, g_{2}\left(r_{1}\right)=1$. 
Proof of (i). It is sufficient to prove that, if $D g=\lambda g, g$ vanishes identically between any two zeros, say $r_{1}^{\prime}, r_{2}^{\prime}$. Apply (9.3), which we have already remarked is applicable, to the $x(t)$ process stopped at these points, assuming that $x(0)=\xi \in\left(r_{1}^{\prime}, r_{2}^{\prime}\right)$. Then (9.3) becomes $0=g(\xi)$, as was to be proved.

Note that, if $D f=\lambda f$, and if $r \in\left(r_{1}, r_{2}\right)$,

$$
f=\frac{f(r)-f\left(r_{1}\right) g_{2}(r)}{g_{1}(r)} g_{1}+f\left(r_{1}\right) g_{2}
$$

because the difference between the two sides is a solution of the differential equation and vanishes at the two points $r_{1}, r$. Thus $g_{1}$ and $g_{2}$ determine the manifold of all solutions.

Proof of (ii). Let $\tau(r)$ be the time a trajectory from $\xi \in\left(r_{1}, r\right)$ first meets an end point of that interval, and let $\tau=\tau\left(r_{2}\right)$. Then, if $x(0)=\xi$, if $g$ is defined by

$$
g=\frac{1-g_{2}(r)}{g_{1}(r)} g_{1}+g_{2}
$$

and if $(9.3)$ is then applied to the $x(t)$ process stopped at $r$,

$$
E\left\{e^{-\lambda r(r)}\right\}=\frac{1-g_{2}(r)}{g_{1}(r)} g_{1}(\xi)+g_{2}(\xi) .
$$

When $r \rightarrow r_{2}$, the left side of this equation converges to $E\left\{e^{-\lambda r}\right\}$. Hence $\left(1-g_{2}\right) / g_{1}$ has a limit $c$ at $r_{2}$, and

$$
E\left\{e^{-\lambda \tau}\right\}=c g_{1}(\xi)+g_{2}(\xi) .
$$

The right side of this equation defines a nontrivial solution of $D f=\lambda f$, of modulus $\leqq 1$.

Proof of (iii). If condition $B\left(r_{2}\right)$ is satisfied, the left side of (9.18) has the limit 1 when $\xi \rightarrow r_{2}$. If condition $C\left(r_{2}\right)$ is satisfied, $\tau$ has a limiting distribution when $\xi \rightarrow r_{2}$, according to the corollary to Theorem 9.7. Thus in both cases the solution we have obtained has a limit at $r_{2}$.

Proof of (iv). According to the corollary to Theorem 9.7, $\tau \rightarrow \infty$ in measure $\left(\xi \rightarrow r_{2}\right)$ when condition $D\left(r_{2}\right)$ is satisfied. Hence the solution in (9.18) has the limit 0 in this case, if $\lambda$ has a positive real part.

Proof of $(\nabla)$. Let ${ }_{2} \tau$ be the time a trajectory from $\xi$ meets $r_{2}$, or $+\infty$ if there is no such meeting, and let ${ }_{2} \tau(r)$ be defined in the same way for the $x(t)$ process stopped at $r$. Then, applying (9.3),

$$
E\left\{e^{-\lambda_{2} r(r)}\right\}=\frac{g_{1}(\xi)}{g_{1}(r)} .
$$

When $r \rightarrow r_{2},{ }_{2} \tau(r) \downarrow_{2} \tau$ with probability 1 , if condition $B\left(r_{2}\right)$ is satisfied. Hence $g_{1}$ must have a finite limit at $r_{2}$ (which we denote by $g_{1}\left(r_{2}\right)$ ) and 


$$
E\left\{e^{-\lambda_{2} r}\right\}=\frac{g_{1}(\xi)}{g_{1}\left(r_{2}\right)} .
$$

We have already seen that there is a limit in (9.18) at $r_{2}$ if condition $B\left(r_{2}\right)$ is satisfied. Hence, under this condition, $g_{1}$ and $g_{2}$, and therefore every solution of the differential equation, has a limit at $r_{2}$.

We now proceed to the proofs of Theorems 9.5, 9.6, 9.7 for complex $\lambda$. We have already remarked that (9.3) of Theorem 9.5 is valid if $\lambda$ has a nonnegative real part. To prove the last assertion of Theorem 9.5 for such a value of $\lambda$, we need only remark that this assertion (say in the case $j=0$, $i=1)$ is an application of (9.3) with $g\left(r_{1}\right)=0, g\left(r_{2}\right)=1$. There is such a solution of the differential equation because, in view of Theorem 9.11(i) as adapted to compact state intervals, if $D f=\lambda f$, and if $f\left(r_{1}\right)=0$ but if $f$ does not vanish identically, we can set $g=f / f\left(r_{2}\right)$. Theorem 9.6 remains true for $\lambda$ with a non-negative real part, that is, the evaluations of Theorem 9.5 remain true for the state interval $\left[r_{1}, r_{2}\right)$ if condition $B\left(r_{2}\right)$ is satisfied. In fact (9.18) and (9.19) represent such evaluations. In view of the results of Theorem 9.11, the derivation of these evaluations in the real case is applicable to the complex case. Theorem 9.7 remains true for $\lambda$ with positive real part, and no change in the proof is required. The theorem is also true, with essentially no change in proof, if $\lambda$ is pure imaginary, under the additional hypothesis that $A\left(r_{2}\right)$ is not satisfied.

\section{BIBLIOGRAPHY}

1. W. Doeblin, Sur certains mouvements aléatoires, C. R. Acad. Sci. Paris vol. 208 (1939) pp. 249-250.

2. J. L. Doob, Stochastic processes, New York, 1953.

3. - Semimartingales and subharmonic functions, Trans. Amer. Math. Soc. vol. 77 (1954) pp. 86-121.

4. W. Feller, Zur Theorie der stochastischen Prozesse (Existenz und Eindeutigkeitssätze), Math. Ann. vol. 113 (1936) pp. 113-160.

5. - The parabolic differential equations and the associated semi-groups of transformations, Ann. of Math. vol. 55 (1952) pp. 468-519.

6. - Diffusion processes in one dimension, Trans. Amer, Math. Soc. vol. 77 (1954) pp. 1-31.

7. Robert Fortet, Les fonctions aleatoires du type de Markoff associees a certaines equations lineaires aux derivees partielles du type parabolique, J. Math. Pures Appl. vol. 22 (1943) pp. 177243.

8. E. Hille, On the integration problem for Fokker-Planck's equation, 10th Congr. of Scandin. Math., Trondheim 1949, pp. 183-194.

9. K. Ito, On stochastic differential equations, Memoirs of the American Mathematical Society, no. 4, 1951.

UNIVERSITY OF ILLINOIS, URBANA, Ill. 